\title{
Localised lateral buckling of partially embedded subsea pipelines with nonlinear
}

\section{soil resistance}

\author{
Zhenkui Wang g,b, G.H.M. van der Heijden ${ }^{\mathrm{b}, *}$ \\ a State Key Laboratory of Hydraulic Engineering Simulation and Safety, Tianjin University, Tianjin 300072, China \\ b Department of Civil, Environmental and Geomatic Engineering, University College London, London WC1E 6BT, UK
}

\section{Corresponding author: G.H.M. van der Heijden, g.heijden@ucl.ac.uk}

\begin{abstract}
Unburied partially embedded subsea pipelines under high temperature conditions tend to relieve their axial compressive force by forming localised lateral buckles. This phenomenon is traditionally studied as a kind of imperfect column buckling problem. We study lateral buckling as a genuinely localised buckling phenomenon governed by a different static instability, with a different critical load. No ad hoc assumptions need to be made. We combine this buckling analysis with a detailed state-of-the-art nonlinear pipe-soil interaction model that accounts for the effect of lateral breakout resistance. This allows us to investigate the effect of initial embedment of subsea pipelines on their load-deflection behaviour. Parameter studies reveal a limit to the temperature difference for safe operation of the pipeline, in the sense that for higher temperature differences a localised buckling mode has lower total energy than the straight unbuckled pipe. Localised lateral buckling may then occur if the pipe is sufficiently imperfect or sufficiently dynamically perturbed.
\end{abstract}

\section{Introduction}

Subsea pipelines are increasingly being required to operate at higher temperatures. The natural tendency is to relieve the resulting high axial load in the pipe wall by localised lateral buckling for unburied subsea pipelines (Bruton et al., 2006). The key uncertainty in lateral buckling design is the lateral soil resistance encountered by the partially embedded pipeline during lateral movement (Dingle et al., 2008). When a pipeline is laid on the seabed, it penetrates partially into the soil due to its self-weight and to other factors, such as the dynamic movement during the laying process, currents/waves and sediment transport (Leckie et al., 2015; Leckie et al., 2016; Randolph and White, 2008; Sumer et al., 2001). This initial embedment has a significant influence on the lateral breakout resistance, which is a key design parameter governing the initiation of lateral buckles (Cheuk et al., 2007). Thus, it is necessary to study the influence of nonlinear lateral soil resistance and breakout resistance on localised lateral buckling.

Much of the past work on pipeline buckling is based on Hobbs's work (Hobbs, 1981; Hobbs, 1984), which itself is based on the very similar work on the buckling of railway tracks (Kerr, 1978). In these works the buckling modes are constructed from three separate zones, a central buckled region, consisting of a column buckling mode, and two adjoining straight regions. Based on this approach, Taylor and co-workers derived an analytical solution to lateral and upheaval buckling for pipelines with initial imperfection (Taylor and Gan, 1986b; Taylor and Tran, 1993; Taylor and Tran, 1996) and analytical solutions for ideal submarine pipelines by considering a deformation-dependent resistance force model (Taylor and Gan, 1986a; Taylor and Gan, 1987). A similar column buckling approach (using slightly different boundary conditions) was used by Croll to study upheaval buckling of pipelines with geometrical imperfections (Croll, 1997).

More recently, Hobbs's method has been adopted by several other studies. Wang and Shi (Shi et al., 2013; Wang et al., 2011) have investigated the upheaval buckling for ideal straight pipelines and for pipelines with prop imperfection on a plastic soft seabed. Moreover, analytical solutions were proposed and compared with finite-element solutions for high-order buckling modes of ideal pipelines and subsea pipelines with a single-arch initial imperfection (Hong et al., 2015b; Liu et al., 2014), which were all based on the classical lateral buckling modes proposed by Hobbs. Karampour and co-workers investigated the interaction between upheaval or lateral buckling and propagation buckling of subsea pipelines (Karampour et al., 2013; 
Karampour and Albermani, 2014; Karampour et al., 2013). There were two limitations in these researches. First, these studies were all based on the assumption of one buckled region and two adjoining regions for the whole pipeline. However, many boundary conditions were introduced when this assumption was employed, which may constrain the lateral deformation of the pipeline. Second, the lateral soil resistance was assumed constant to simplify the theoretical results.

For the central buckled region Hobbs takes a sine wave and introduces decay by means of imperfections. It is good to point out, however, that for this type of beam-on-foundation problems there exists a mechanism for genuine localised buckling that does not require one to make such ad hoc approximations. In this paper we discuss this localised buckling in some detail, show how localised solutions can be conveniently and reliably computed and compare results with Hobbs's. We also use a realistic soil resistance model, which leads to differences in the load-deflection curves.

Localised buckling is quite different from (Euler) column buckling. It is described by a so-called Hamiltonian-Hopf bifurcation rather than the pitchfork bifurcation of column buckling. An important consequence is that unlike the critical load for column buckling, which depends strongly (quadratically) on the length of the structure, the critical load for localised buckling does not depend on this length (although the structure of course has to be long enough to support a localised buckle). Importantly, the critical load for localised buckling is found to be lower than that for Euler buckling. Although this critical load is generally not reached and localised deflection is initiated by imperfections or perturbations, this critical load still provides a useful reference load. For sufficiently long slender structures, localised buckling is also energetically much more favourable than periodic buckling into a (large) number of half sine waves (Hunt et al., 1989).

The advantage of describing localised buckling by means of branches of solutions emanating from a Hamiltonian-Hopf bifurcation is that these solutions come with simple analytical estimates (in terms of the linear system parameters) for the 'wavelength' of the buckling pattern (e.g., the length of pipe in the central buckle) as well as the decay rate of successive buckles, without the need for some kind of damping or imperfections. The theory also predicts both symmetric and antisymmetric buckling modes (as also constructed in (Hobbs, 1984)). This is simply dictated by the symmetry properties of the beam equilibrium equation.

One of the few papers that do not make Hobbs's assumption of separate buckled and adjoining regions is that of Zhu et al. (Zhu et al., 2015). They therefore compute true localised solutions, although they do not discuss the different mechanism giving rise to this localised buckling. Indeed, they impose classical boundary conditions of Euler-type buckling that do not maintain localisation as parameters of the system (e.g., the temperature difference) are varied. They also only obtain the symmetric and not the anti-symmetric mode and don't compute the critical load for localised buckling.

As to the soil modelling, different soil resistance models have been incorporated into the localised lateral buckling problem. Lagrange and Averbuch (Lagrange and Averbuch, 2012) have studied the periodic solutions of a strut on a nonlinear elastic Winkler-type foundation with imperfection in the form of a sine shape. The nonlinear restoring force of the foundation was either a bi-linear or an exponential profile. Piecewise solution theory was used to solve the governing equations for the bilinear restoring force. Piecewise solution theory was also employed by Karampour et al. (Karampour et al., 2015) to obtain the analytical solution to lateral buckling of pipelines with a softening foundation. Zhu et al. (Zhu et al., 2015) proposed a new approach for determining the nonlinear behaviour of pipelines under thermal loading. However, the lateral soil resistance was modelled by a hyperbolic function in their research, which didn't consider the influence of breakout resistance on the localised lateral buckling of unburied subsea pipelines. Zeng and Duan (Zeng and Duan, 2014) studied lateral buckling of partially embedded submarine pipelines with the pipeline modelled as an axial compressive beam supported by lateral distributed nonlinear springs. The nonlinear springs take the soil berm effect into account in the horizontal plane. But this nonlinear lateral resistance is only applicable in cases of small lateral displacement. In reality the amplitude of localised lateral buckling for unburied subsea pipelines may exceed ten pipeline diameters (White and Cheuk, 2008). Therefore, in this paper we introduce the nonlinear lateral soil resistance model proposed by Chatterjee et al. (Chatterjee et al., 2012), which can be applied in cases of large-amplitude lateral movement. The model is described in detail in the following section.

The purpose of this paper is therefore twofold. (i) We show that thermal pipeline buckling is well described by genuinely localised (and exponentially decaying) solutions that bifurcate from the straight pipe at a critical temperature. We explore the 
consequences of this localised buckling phenomenon without making any additional assumptions and pick up a few simple analytical results that may be useful as design formulae. (ii) We employ a realistic state-of-the-art nonlinear pipe-soil interaction model to compute load-deflection curves that take into account lateral breakout resistance due to pipe embedment.

The rest of the paper is organised as follows. In Section 2 we present the mathematical modelling of lateral pipeline buckling. We use a beam-on-foundation model for the lateral deflection of the pipe and compatibility between axial and lateral deformation to derive a relationship between the compressive axial force in the pipe and the temperature difference between the pipe and its environment. The soil resistance model is discussed in detail in Section 2.3. The method used for computing localised solutions is explained in Section 2.4. It uses a shooting method that exploits the symmetry properties of the equilibrium equation. Parameter studies are carried out by numerical continuation (path following) techniques in Section 3. We also compare our solutions with those of Hobbs. Stability of the localised solutions is analysed by computing the total energy of the pipeline. A critical temperature is identified beyond which imperfection-driven localised lateral buckling may be expected. Furthermore, the influence of breakout resistance on localised lateral buckling is studied and discussed. Section 4 closes this study with some conclusions.

\section{Problem modelling}

\subsection{Pipeline buckling under thermal loads}

We imagine a pipeline laid on a horizontal surface (the seabed) and subjected to a temperature difference $T_{0}$ between the fluid flowing inside the pipe and the environment. If the ends of the pipe are unrestrained then under an increase of the temperature difference the pipe will expand axially. This expansion will be resisted by friction between pipe and seabed (and surrounding soil). If the soil resistance for axial movement is constant, say $f_{A}$, then a compressive force will build up in the pipe, which will increase linearly with the distance from the freely-expanding end. At some point this compressive force is sufficient to halt further expansion of the central segment of the pipe. Thus an immobilised segment spreads from the centre of the pipe. The end points of this segment are called virtual anchor points. Between these points the compressive force in the pipe is equal to the force in a pipe with fixed ends under the same thermal load. Within the range of linear elastic response this compressive force can be written as

$$
P_{0}=E A \alpha T_{0}
$$

where $E$ is the elastic modulus. $A$ is the cross-sectional area of the pipeline and $\alpha$ is the coefficient of linear thermal expansion. Immobilisation will only occur if this compressive force is attained, which in the present scenario will only be the case if the length of the pipe is larger than $2 l_{i}$, where

$$
l_{i}=E A \alpha T_{0} / f_{A}
$$

Under increasing temperature difference, the compressive force $P_{0}$ increases and at some point buckling may be initiated. As stated in the Introduction, for a sufficiently long pipe this will be localised buckling, with exponentially decaying deflection. For a pipe without imperfections we expect this buckling to occur in the centre of the pipe. Here we shall assume this buckling to be lateral, i.e., horizontal, against the resistance of the surrounding soil, rather than vertical, against gravity. For normal coefficients of friction, the lateral mode occurs at a lower axial load than the vertical mode (Hobbs, 1984). Lateral buckling is therefore dominant, except in cases where lateral deflection is prevented, such as for pipes laid in a trench.

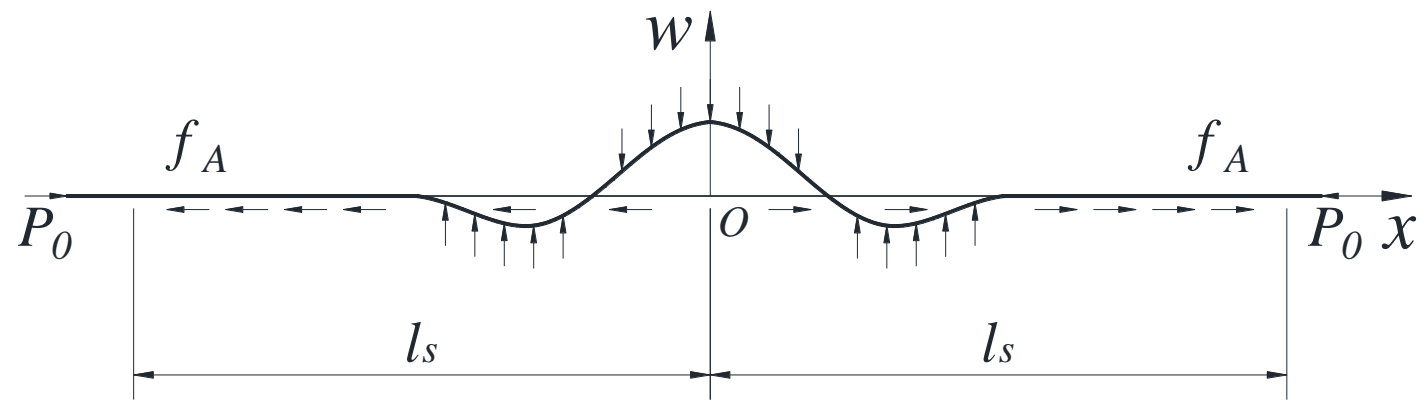


Fig. 1 Configuration and load distribution of localised lateral buckling.

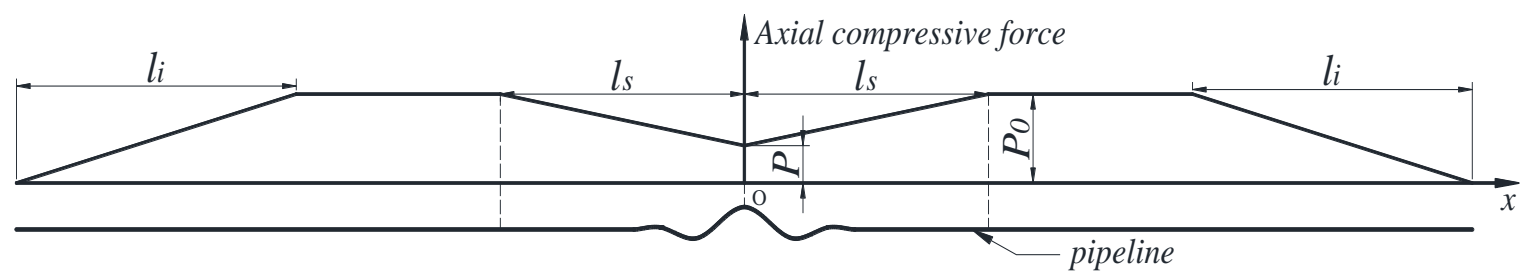

Fig. 2 Axial compressive force distribution of localised lateral buckling.

In the buckling process a small central segment of the pipe will mobilise. The same scenario as described above applies, but now in reverse. Thus, as pipe feeds into the buckle the compressive force in the pipe drops, pulling more pipe into the buckle. This feed-in will be halted at two more virtual anchor points at compressive force $P_{0}$ bounding the mobilised region. Fig. 2 shows the feed-in region within the larger immobilised pipe segment of length $l_{s}$ with the localised buckle and the typical compressive force variation. $l_{s}$ is sometimes called the slip-length. In practice multiple (independent) localised buckles may form in the immobilised pipe segment, especially if it is long. In the following we present a theory for a single localised buckle that applies to each such buckle individually.

\subsection{Governing equations and deformational compatibility}

The partially embedded pipeline subject to high temperature is idealised as an axial compressive Euler-Bernoulli beam supported by distributed springs on both sides in the horizontal plane. The distributed springs simulate the nonlinear lateral soil resistance, which is provided by the soil foundation when the partially embedded pipeline deforms laterally during the process of localised lateral buckling. Fig. 1 illustrates the typical configuration of lateral buckling for a subsea pipeline resting on the seabed. Note that by symmetry we need only consider half the length of the pipe $\left(0 \leq x \leq l_{s}\right)$. Thus we have the following equation for the lateral deformation of the pipeline:

$$
E I \frac{d^{4} w}{d x^{4}}+\bar{P} \frac{d^{2} w}{d x^{2}}+h=0
$$

where $w$ is the lateral displacement, $E I$ is the bending stiffness, $\bar{P}$ is the axial compressive force and $h$ is the nonlinear lateral soil resistance. We assume that $\bar{P}$ has the profile sketched in Fig. 2, i.e., $\bar{P}=P$ at the centre of the pipe and $\bar{P}=P_{0}$ at the end of the mobilised buckling region. Boundary conditions for Eq. (3), which must support localised solutions as in Fig. 1, will be discussed in detail in Section 2.4.

Axial deformation of the pipeline is governed by the equation

$$
E A \frac{d^{2} u}{d x^{2}}=f_{A}
$$

For the axial soil resistance (a force per unit length) we can write

$$
f_{A}=\mu_{A} W_{\text {pipe }}
$$

where $\mu_{A}$ is the axial friction coefficient between pipeline and seabed and $W_{\text {pipe }}$ is the submerged weight per unit length of the pipeline. Eq. (4) is solved subject to the slip-length boundary conditions (Taylor and Gan, 1986b)

$$
\left\{\begin{array}{c}
u\left(l_{\mathrm{s}}\right)=0 \\
\frac{d u}{d x}\left(l_{\mathrm{s}}\right)=0
\end{array}\right.
$$

giving for the axial displacement

$$
u(x)=\frac{f_{A}}{2 E A}\left(x-l_{\mathrm{s}}\right)^{2}
$$

This result will be used later when computing the total potential energy of a localised pipe solution.

We now use compatibility between axial and lateral deformation in the immobilised region $0 \leq x \leq l_{s}$ to derive a relationship between the axial compressive force $P$ at the centre of the pipe and the temperature difference $T_{0}$. Compatibility can be expressed as 


$$
u_{1}=u_{2}
$$

$u_{1}$ is the length of axial expansion within the pipeline section $0<x<l_{s}$ due to high temperature. $u_{2}$ is the geometric shortening, which allows for the additional length introduced by the lateral displacement. Eq. (8) simply states that, since there are virtual anchor points at distance $l_{\mathrm{s}}$ from the centre of the pipe, the extra length of pipe in the buckle must come from axial expansion of the mobilised section of pipe.

We have

$$
\mathrm{u}_{1}=\int_{0}^{l_{s}} \frac{\Delta \bar{P}(x)}{E A} d x
$$

Here $\Delta \bar{P}(x)$ is the amount of decrease of axial compressive force along the pipeline after the pipeline buckles, given by

$$
\Delta \bar{P}(x)=f_{A}\left(l_{s}-x\right)
$$

Thus, we find

$$
u_{1}=\frac{f_{A} l_{s}^{2}}{2 E A}
$$

For $u_{2}$ we have

$$
u_{2}=\frac{1}{2} \int_{0}^{l_{s}}\left(\frac{d w}{d x}\right)^{2} d x
$$

Thus, combining Eq. (8) and Eq. (11), we obtain the following equation

$$
l_{s}=\sqrt{\frac{2 E A u_{2}}{f_{A}}}
$$

By axial force balance, we have

$$
P_{0}-P=f_{A} l_{s}
$$

Combining Eq. (1), Eq. (13) and Eq. (14), we finally obtain

$$
T_{0}=\frac{\left(P+\sqrt{2 E A u_{2} f_{A}}\right)}{E A \alpha}
$$

For consistency we require $l_{s}$ to be larger than the length of pipe in the localised buckle. Since there is no a priori guarantee that $l_{s}$ as computed from Eq. (13) satisfies this condition, we need to check any computed solutions for acceptability.

\subsection{Soil resistance}

For the study of the influence of nonlinear lateral soil resistance on pipeline localised lateral buckling, the selection of a suitable and practical lateral soil resistance model is of great importance. Present industry practice estimates the soil resistance with the Coulomb friction model, which expresses the lateral soil resistance as the product of effective submerged weight of the pipeline and a friction coefficient lying in the range of 0.2-0.8 (Lambrakos, 1985; Lyons, 1973; Wagner et al., 1989). This means that the lateral soil resistance is regarded as constant, which has been employed to study the analytical solutions of lateral buckling for subsea pipelines by many researchers (Karampour et al., 2013; Hobbs, 1984; Hong et al., 2015a; Hong et al., 2015b; Li et al., 2016; Liu et al., 2014; Taylor and Gan, 1986b). Consistent deformation-dependent resistance force models were incorporated into the analyses of lateral buckling by Taylor and Gan (Taylor and Gan, 1986a) and Zhu et al. (Zhu et al., 2015). Bi-linear and tri-linear restoring force models were employed to obtain the analytical solutions of an axially loaded strut (Lagrange and Averbuch, 2012) and an axially loaded subsea pipeline (Karampour et al., 2015), respectively. Furthermore, a nonlinear lateral soil resistance model, which contained cubic and quintic nonlinear terms, was used by Zeng and Duan (Zeng and Duan, 2014) to obtain localised solutions with an intricate bifurcation structure. However, this model is only suitable for very small lateral displacements $w$ because of the assumed large positive slope $d h / d w$ for large $w$, which is not realistic for pipelines.

The aforementioned lateral soil resistance models are not practical for lateral buckling of partially embedded pipelines, which undergo large-amplitude lateral displacement and are subject to nonlinear lateral soil resistance. For the localised lateral buckling of partially embedded pipelines, White and Cheuk presented some nonlinear force-displacement models, which took account of the effects of pipe initial penetration and soil berms based on experimental data, such as the tri-linear lateral 
resistance model (White and Cheuk, 2008) and the nonlinear lateral soil resistance model expressed by exponential functions (Chatterjee et al., 2012). The nonlinear lateral soil resistance model proposed by Chatterjee et al. (Chatterjee et al., 2012) is chosen in this paper because it is suitable for large-amplitude lateral displacements. The lateral soil resistance for different breakout resistances shown in Fig. 3 can be obtained by the following expression

$$
\begin{gathered}
h=\frac{w}{|w|}\left(H_{\text {brk }}\left(1-\mathrm{e}^{-a_{1} *\left(\frac{|w|}{D}\right)^{a_{2}}}\right)+\left(H_{\text {res }}-H_{\text {brk }}\right)\left(1-\mathrm{e}^{-a_{3} *\left(\frac{|w|}{D}\right)^{a_{4}}}\right)\right) \\
H_{\text {brk }}=\mu_{\text {brk }} W_{\text {pipe }}, H_{\text {res }}=\mu_{\text {res }} W_{\text {pipe }}
\end{gathered}
$$

Here, $H_{b r k}$ is the breakout resistance and $H_{r e s}$ the residual resistance, $\mu_{b r k}$ is the friction coefficient corresponding to breakout resistance, $\mu_{\text {res }}$ is the friction coefficient corresponding to residual resistance and $D$ is the external diameter of the pipe.

The first term of Eq. (16) only controls the initial mobilisation of the breakout resistance, while the second term provides a smooth exponential transition from the breakout resistance to the residual resistance. The values of coefficients $a_{1}, a_{2}$ and $a_{4}$ remain essentially constant for all values of initial embedment and pipe weight, and, following (Chatterjee et al., 2012), are assigned to be $25,0.5$ and 1.5 , respectively, for all cases. However, the value of $a_{3}$, which determines the distance required to mobilise the steady resistance, changes with pipe weight and initial embedment. For any given initial embedment, the value of $a_{3}$ is given by

$$
a_{3}=c\left(\frac{W_{\text {pipe }}}{V_{\max }}\right)+d
$$

The values of $\mathrm{c}$ and $\mathrm{d}$ for different initial embedment are given by

$$
\begin{aligned}
& c=8.2 \frac{w_{\text {init }}}{D}-4.9 \\
& d=-5.8 \frac{w_{\text {init }}}{D}+4.5
\end{aligned}
$$

$V_{\text {max }}$ is the vertical bearing capacity and $w_{\text {init }}$ is the initial embedment of the pipeline. The values of $V_{\text {max }}$ and $w_{\text {init }}$ considered in this paper are $5 W_{\text {pipe }}$ and $0.3 \mathrm{D}$, respectively. Thus, the value of $a_{3}$ obtained by Eq. (18) is 2.272 , which is used in this study.

Of all the parameters introduced above only $\mu_{b r k}$ is varied in this paper; all other parameters in the nonlinear soil resistance model are fixed at the above values.

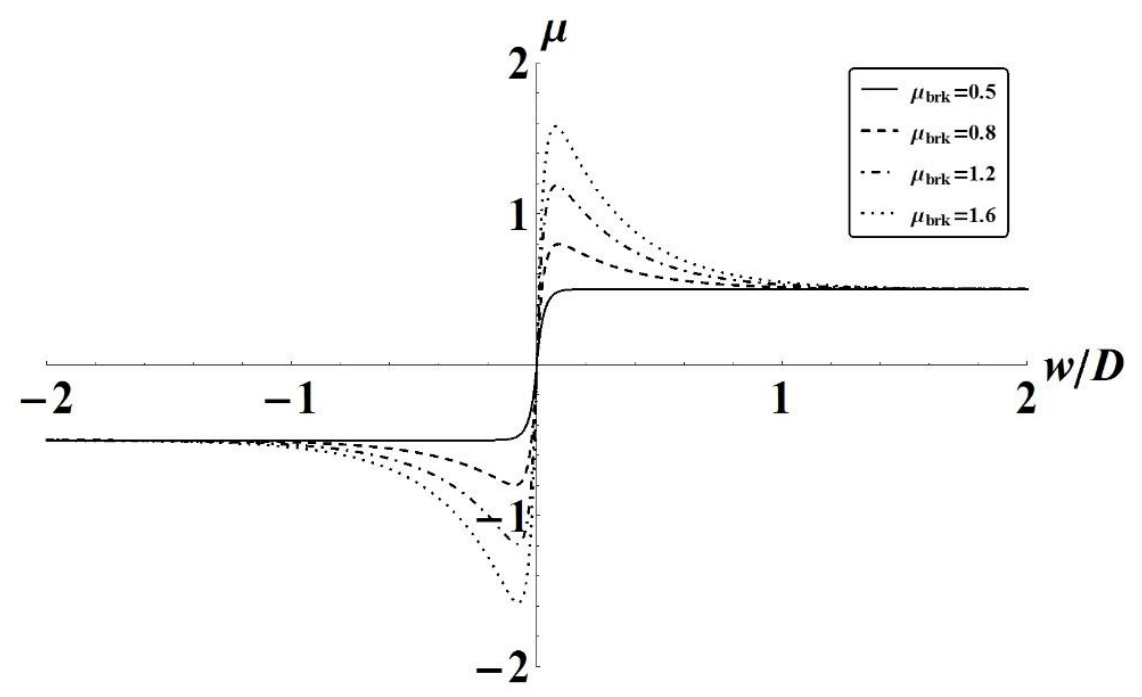

Fig. 3 Nonlinear lateral soil resistance model at different values of the breakout resistance coefficient $\mu_{b r k}$. 


\subsection{Localised buckling}

Here we discuss the localised solutions of Eq. (3). We now make the assumption that the axial compressive force is constant in the buckled region and equal to the force at the center of the buckle, i.e., $\bar{P}=P$. The same approximation was made by Hobbs (Hobbs, 1984). It is useful to rewrite the fourth-order Eq. (3) as an equivalent four-dimensional system of first-order equations $\left(w=w_{1}\right)$ :

$$
\left\{\begin{array}{c}
\frac{d w_{1}}{d x}=w_{2} \\
\frac{d w_{2}}{d x}=w_{3} \\
\frac{d w_{3}}{d x}=w_{4} \\
\frac{d w_{4}}{d x}=-\frac{1}{E I}\left(P w_{3}+h\right)
\end{array}\right.
$$

Solutions of Eq. (21) are orbits in a four-dimensional phase space with coordinates $\left(w_{1}, w_{2}, w_{3}, w_{4}\right)$. The straight pipe solution is represented by the fixed point $\mathrm{j}=(0,0,0,0)$. The eigenvalues of the fixed point are:

$$
\pm i \sqrt{\frac{P \pm \sqrt{P^{2}-4 E I k}}{2 E I}}
$$

where $\mathrm{k}=\left(\frac{\mathrm{dh}}{\mathrm{dw}}\right)_{w=0}$. We conclude that at the critical load $P=P_{c r}$ with

$$
P_{c r}=2 \sqrt{k E I}
$$

the eigenvalues change from a quadruple of complex eigenvalues to two complex conjugate pairs of imaginary eigenvalues (see Fig. 4). This is called a Hamiltonian-Hopf bifurcation (Hunt et al., 1989; van der Heijden et al., 1998) and marks the loss of stability of the straight solution. For comparison, the critical load for buckling of a pinned-pinned beam into a pattern of $n$ half sine waves is

$$
P_{\text {cr,periodic }}=\frac{n^{2} \pi^{2} E I}{L^{2}}+\frac{k L^{2}}{n^{2} \pi^{2}}
$$

It is straightforward to show that $P_{c r} \leq P_{c r, p e r i o d i c}$ for all $n$.

The symmetry and multiplicity of bifurcating solutions is governed by the symmetry of the system of equations. We have the following two reversing symmetries (i.e., the equations are invariant under the following simultaneous sign changes)

$$
\begin{aligned}
& R_{1}: x \rightarrow-x, \quad\left(w_{1}, w_{2}, w_{3}, w_{4}\right) \rightarrow\left(w_{1},-w_{2}, w_{3},-w_{4}\right) \\
& R_{2}: x \rightarrow-x, \quad\left(w_{1}, w_{2}, w_{3}, w_{4}\right) \rightarrow\left(-w_{1}, w_{2},-w_{3}, w_{4}\right)
\end{aligned}
$$

It is well-known that among the solutions bifurcating from the trivial straight solution into the region of the complex quadruple of eigenvalues (here for $P<P_{c r}$ ) are so-called homoclinic orbits that leave the unstable fixed point in the plane spanned by the eigenvectors corresponding to the unstable eigenvalues (with positive real part), make a large excursion in the phase space and then return to the fixed point in the plane spanned by the eigenvectors corresponding to the stable eigenvalues (with negative real part) (Champneys and Spence, 1993; van der Heijden et al., 1998). These solutions thus approach the straight solution in both limits $x \rightarrow \pm \infty$ and are therefore also called localised solutions. Because of the above reversing symmetries, both a symmetric $\left(R_{1}\right.$-reversible) and an anti-symmetric $\left(R_{2}\right.$-reversible) solution bifurcates. Half these localised solutions are shown in Fig. 5-a, while the corresponding half orbits in (a two-dimensional projection of) the phase space are shown in Fig. 5-b. Note that the homoclinic orbits spiral out of (and back into) the fixed point because of the complex eigenvalues.

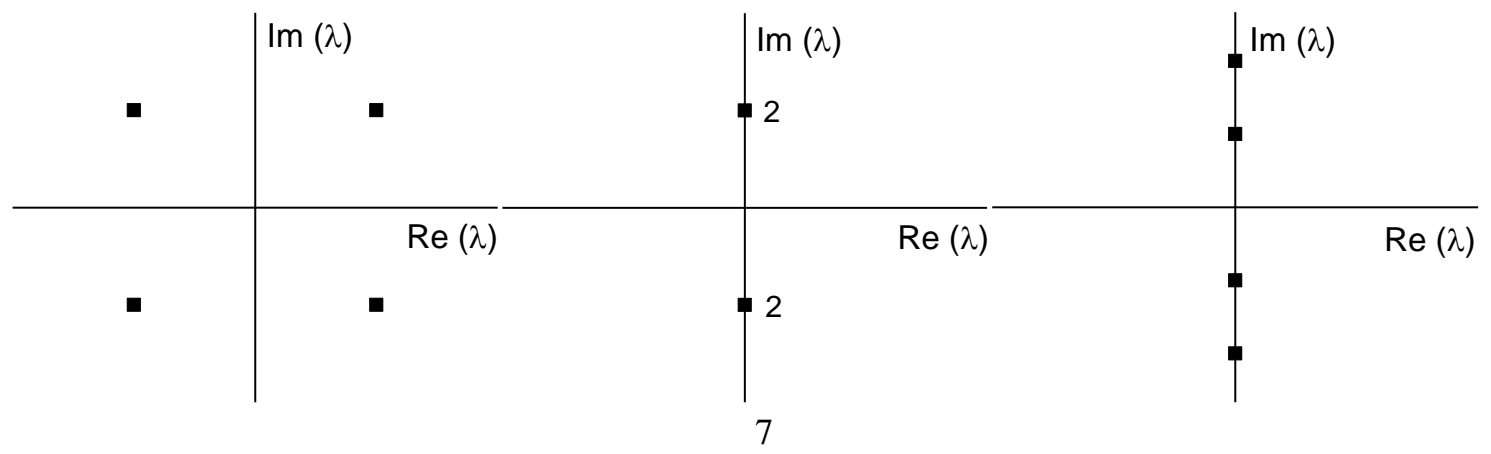


Fig. 4 The behaviour of eigenvalues at the Hamiltonian-Hopf bifurcation. (a) $P<P_{c r}$. (b) $P=P_{c r}$. (c) $P>P_{c r}$.

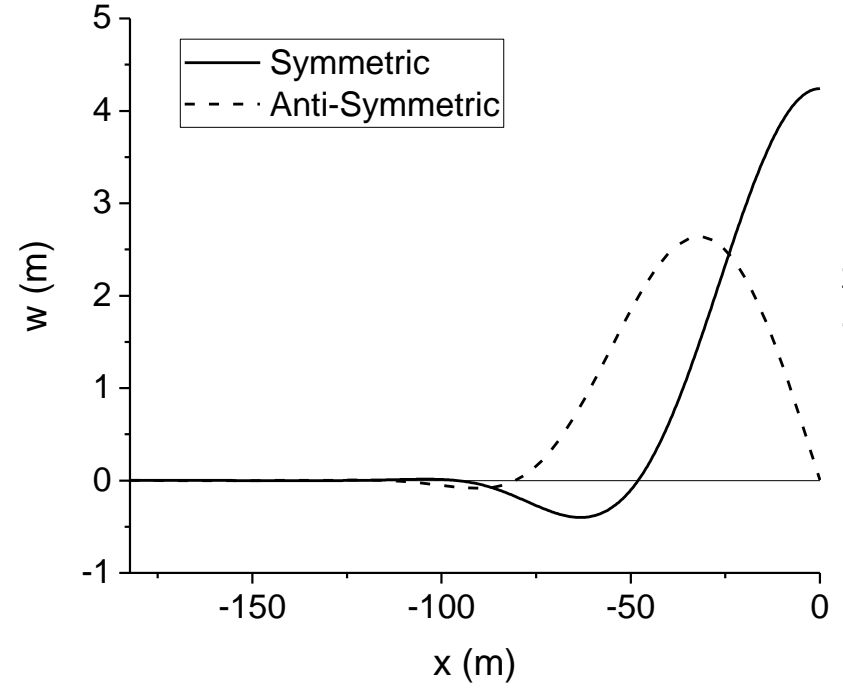

(a)

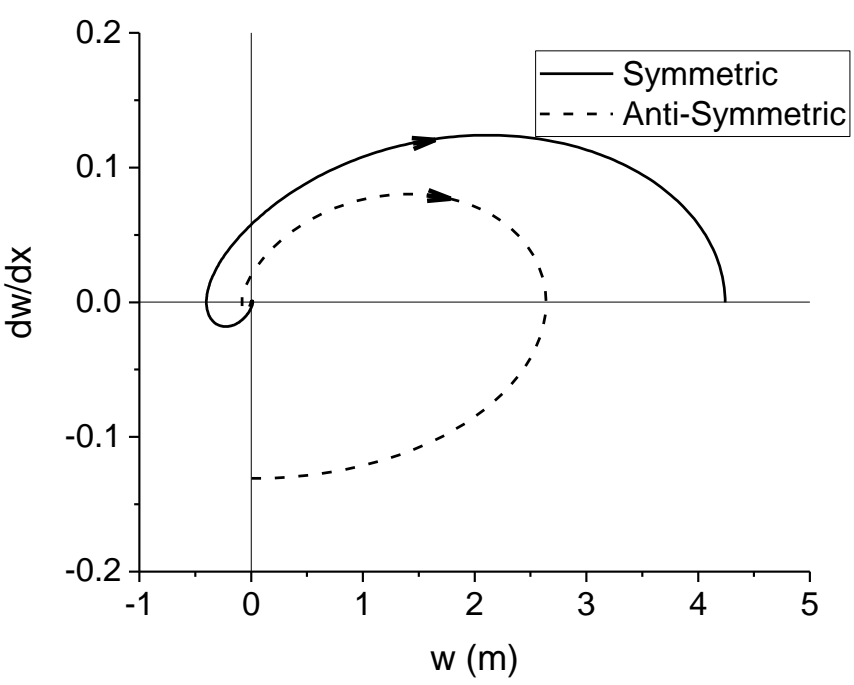

(b)

Fig. 5 Typical solution obtained by the shooting method. (a) The deformed shapes. (b) The homoclinic orbits in phase space.

$$
\mu_{b r k}=0.8 . P=1.4 \mathrm{MN} \text {. }
$$

For later reference we also record here that for $P<P_{c r}$ the eigenvalues in Eq. (22) can be written as $\pm \lambda \pm i \omega$, with real $\lambda$ and $\omega$ given by

$$
\lambda=\frac{\sqrt{2 \sqrt{E I k}-P}}{2 \sqrt{E I}}, \quad \omega=\frac{\sqrt{2 \sqrt{E I k}+P}}{2 \sqrt{E I}}
$$

Expansion about the critical load gives

$$
\lambda=\frac{\sqrt{P_{c r}-P}}{2 \sqrt{E I}}, \quad \omega=\sqrt[4]{\frac{k}{E I}}-\frac{P_{c r}-P}{4 \sqrt{2 E I P_{c r}}}+\mathrm{O}\left(\left(P_{c r}-P\right)^{2}\right)
$$

\section{Analysis of localised solutions}

\subsection{Numerical computation of localised solutions}

For $P<P_{c r}$ we compute approximate (half) homoclinic solutions as in Fig. 5-a by formulating a shooting method on a truncated $x$ interval $[-L, 0]$. Here $L$, the half length of the homoclinic solution, is chosen large enough that the solution is well-localised in the sense that it is very nearly decayed to the trivial straight solution $j$ at $x=-L$. Thus we specify initial conditions

$$
w(-L)=j+\varepsilon\left(v_{1} \cos \delta+v_{2} \sin \delta\right)
$$

where $v_{1} \pm i v_{2}$ are eigenvectors corresponding to eigenvalues $\lambda \pm i \omega$ of $j . \varepsilon$ is a small constant, while $\delta$ and $L$ are two shooting parameters that are initially guessed and iteratively updated by means of two boundary conditions. The parameter $\delta$ is the angle about the fixed point where the outward spiraling homoclinic orbit cuts the circle of radius $\varepsilon$ around the fixed point in the unstable eigenspace. For the required two boundary conditions we take advantage of the symmetry properties in Eq. (25) and Eq. (26). We impose

$$
\left\{\begin{array}{l}
w_{2}(0)=0 \\
w_{4}(0)=0
\end{array}\right.
$$

for symmetric solutions and 


$$
\left\{\begin{array}{l}
w_{1}(0)=0 \\
w_{3}(0)=0
\end{array}\right.
$$

for anti-symmetric solutions (see Fig. 5). The half orbits thus computed can readily be turned into full orbits by appropriate reflection according to $R_{1}$ or $R_{2}$. Shooting over half the interval is numerically better behaved than shooting back into the neighbourhood of the unstable fixed point. The constant $\varepsilon$ sets the scale of $L$. We choose $\varepsilon=10^{-5}$, which is found to yield well-localised solutions. The parameters used in this study are presented in Table 1. For these parameters and the additional choice $\mu_{b r k}=0.8$, we have $k=139512.62 \mathrm{~N} / \mathrm{m}$. For the case $P=1.4 \mathrm{MN}$, as shown in Fig. 5, the values of $\delta$ and $L$ are listed in Table 2. From Eq. (13) we also compute $l_{s}=1204.945 \mathrm{~m}$ for the symmetric solution and $l_{s}=1192.791 \mathrm{~m}$ for the anti-symmetric solution, noting that both are larger than $L$, as required. The eigenvalues corresponding to the unstable manifold of the origin are $\lambda \pm i \omega$, where $\lambda=0.097295, \omega=0.108250$, and we use

$$
\begin{gathered}
v_{1}=(0.989336,0.096317,0.002234,-0.002478) \\
v_{2}=(0,0.107178,0.020869,0.001790)
\end{gathered}
$$

in Eq. (29).

Fig. 6 shows a bifurcation diagram obtained by varying the parameter $P . w_{m}$ is the maximum lateral deflection. The plot in Fig. 6 confirms that the post-buckling localised solutions exist for loads smaller than the critical load $P_{c r}$, which, from Eq. (23), is $P_{c r}=13.171288 \mathrm{MN}$, i.e., the localised solutions bifurcate subcritically. Such subcritical bifurcations are wellknown to give rise to imperfection sensitivity (Hutchinson and Koiter, 1970). Fig. 7 shows a solution for $P=12.8 \mathrm{MN}$, close to the critical load, illustrating the oscillatory decay of the (small-amplitude) localised solutions governed by the eigenvalues $\pm \lambda \pm i \omega$. The wavelength of the solution, i.e., the distance between two successive minima, is almost constant and agrees very well with the period $2 \pi / \omega=43.4765 \mathrm{~m}$, while the decay rate $w_{m 2} / w_{m 1}=0.873575$ is well approximated by $e^{-2 \pi \lambda}=$ 0.897118 (the agreement would be even better for values of $P$ closer to $P_{c r}$ ). The asymptotic result in Eq. (28) shows that the decay rate depends on the distance from the critical load. The solution increasingly localises as the load $P$ is reduced. For a (half) solution to be called localised the length $L$ has to be larger than the localisation length $L_{l}$ defined by

$$
L_{l}=\frac{1}{\lambda}=2 \sqrt{E I /\left(P_{c r}-P\right)}
$$

Typical loads $P$ in pipelines stay well away from the critical load $P_{c r}$, and therefore in practice only a few oscillations (lobes) are visible and the solution is very well localised, as in Fig. 5-a. The fact that localisation is observed at values of $P$ much lower than $P_{c r}$, where the straight pipe is stable, is usually explained by inevitable imperfections and dynamical disturbances and will be discussed by means of an energy analysis in Section 3.3. We also note that for the solution of Fig. $7, l_{\mathrm{s}}=7.898095$, which is much smaller than the length of rod in the localised buckle, so this solution does not satisfy the compatibility condition Eq. (8). All other solutions presented in this paper do satisfy this condition.

Table 1 Design parameters.

\begin{tabular}{ccc}
\hline Parameters & Values & Unit \\
\hline External diameter $D$ & 650 & $\mathrm{Mm}$ \\
Wall thickness $t$ & 15 & $\mathrm{Mm}$ \\
Elastic modulus $E$ & 206 & $\mathrm{GPa}$ \\
Coefficient of thermal expansion $\alpha$ & $1.1 \times 10^{-5}$ & $/{ }^{\circ} \mathrm{C}$ \\
Poisson ratio $v$ & 0.3 & --- \\
Lateral residual friction coefficient $\mu_{\text {res }}$ & 0.5 & --- \\
Axial friction coefficient $\mu_{A}$ & 0.5 & --- \\
Submerged weight $W_{\text {pipe }}$ & 3800 & $\mathrm{~N} / \mathrm{m}$ \\
\hline
\end{tabular}

Table 2 Shooting parameters. $P=1.4 \mathrm{MN}$.

\begin{tabular}{lll}
\hline Reversible under & $\delta$ & $\mathrm{L}(\mathrm{m})$ \\
\hline
\end{tabular}




\begin{tabular}{lll}
\hline$R_{1}$ & 1.378680 & 182.335638 \\
$R_{2}$ & 3.180571 & 188.532284 \\
\hline
\end{tabular}

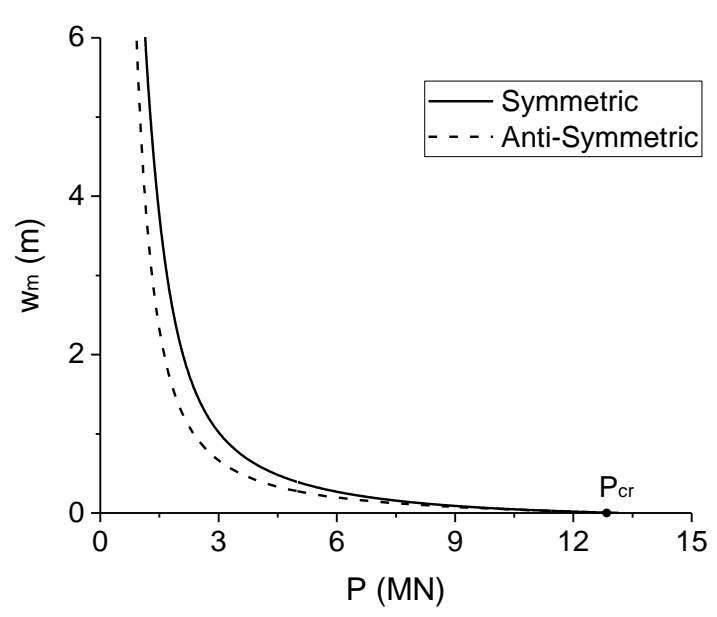

Fig. 6 Bifurcation diagram with two branches of homoclinic orbits bifurcating subcritically at the critical load $P_{c r}$.

$$
\mu_{b r k}=0.8 \text {. }
$$

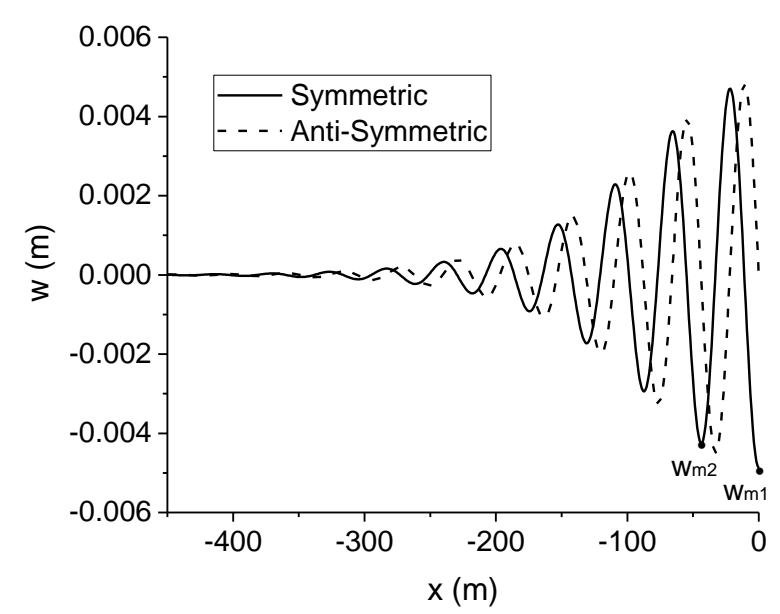

Fig. 7 The deformed shapes close to the critical point. $P=12.8 \mathrm{MN} . \mu_{b r k}=0.8$.

\subsection{Comparison with results in the literature}

In this section, the localised lateral buckling results of unburied partially embedded subsea pipelines with nonlinear soil resistance obtained through using the method described in previous section are compared with the results of Hobbs (Hobbs, 1984). In Hobbs's study, four lateral buckling modes are proposed, whose analytical solutions are obtained based on the assumption of constant lateral soil resistance. The deformed shapes and the buckling paths are shown in Fig. 8 and Fig. 9, respectively. Only half deformed shapes of the buckled pipeline are plotted in Fig. 8 due to symmetry and anti-symmetry. In Hobbs's analysis, mode 1 and mode 3 are symmetric modes, which are employed to compare with the symmetric solution. Mode 2 and mode 4 are anti-symmetric modes, which are employed to compare with the anti-symmetric solution. In Fig. 8$\mathrm{a}$, it is clear that the deformed shape of mode 3 is similar to the deformed shape of our symmetric solution. However, the deformed shape of mode 1 has a big difference with the deformed shape of the symmetric solution. The maximum lateral displacement of mode 3 is a bit larger than that predicted by the method in this paper, both of which are much smaller than that of mode 1, because only one lobe exists in mode 1, which constrains the lateral deformation of the buckled pipeline. The buckling path of mode 3 coincides well with that predicted in this paper, which is quite different from that of mode 1, as shown in Fig. 9-a. The minimum critical temperature of mode 1 is larger than that of the other two cases. As for anti-symmetric solutions, the deformed shape and the buckling path of mode 4 coincide better than those of mode 2 . 
The reason for the difference is that Hobbs assumed that there was no lateral deflection in the adjoining regions outside the assumed lobes, which means that the pipeline can only deform axially in adjoining regions and the axial compressive force in the buckled region is overestimated, especially for mode 1, as shown in Fig. 10-a. The overestimated axial compressive force results in overestimated displacement in the buckled region. Also, this assumption will change the deformed shape of the buckled pipeline. For mode 1, there is only one primary lobe within the buckled region. For mode 3 , a secondary lobe exists adjacent to the primary lobe. However, there is a much smaller lobe adjacent to the secondary lobe for the localised lateral buckling of partially embedded pipelines, which is shown in Fig. 8-a. So the assumption of mode 1 and mode 3 constrains the lateral deformation of partially embedded subsea pipelines. In the analysis presented in this paper, no assumption is made about the lateral deformation in the localised buckled region (recall from the previous section that the decay rate of lobe amplitudes is governed by the real part $\lambda$ of the eigenvalues of the homoclinic orbit). For anti-symmetric solution, similar conclusions can be obtained.

Our detailed analysis shows that it is feasible and effective to obtain the deformed shape and the buckling path by using the assumption of mode 3 for symmetric solution and mode 4 for anti-symmetric solution on condition that the lateral soil resistance stays constant. However, Hobbs's method cannot be applied to localised lateral buckling when the lateral soil resistance is nonlinear as is the case for partially embedded subsea pipelines.

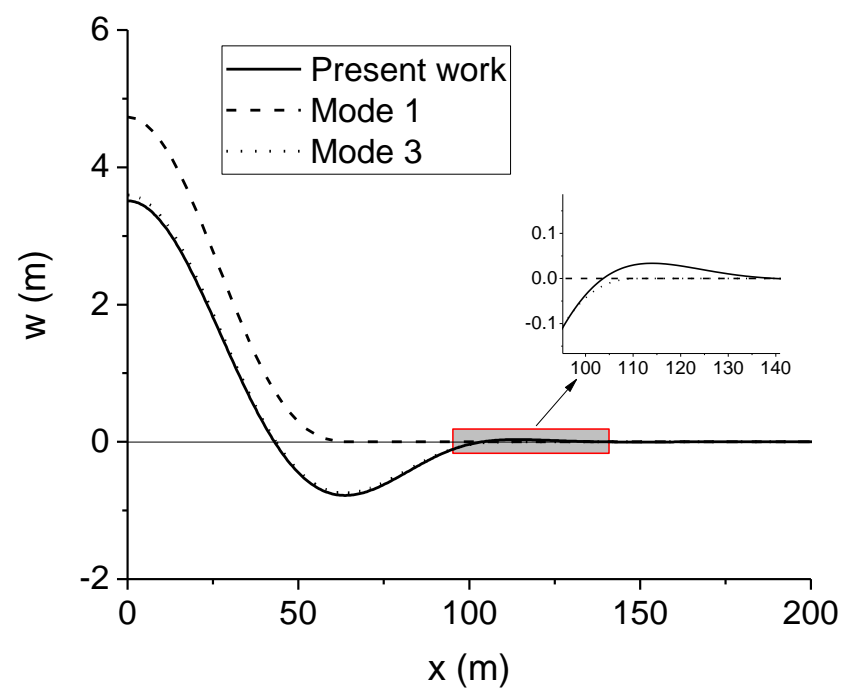

(a)

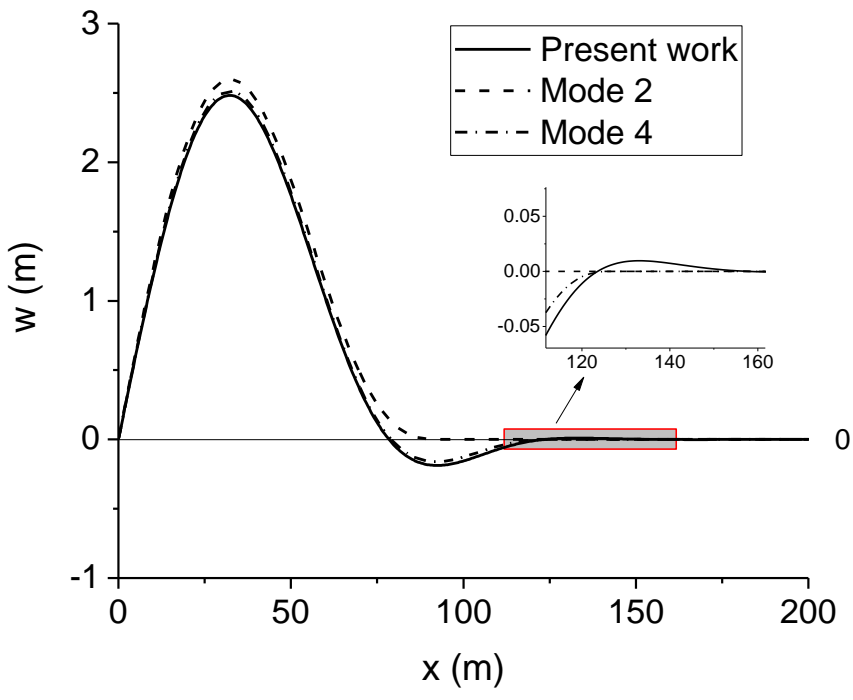

(b)

Fig. 8 Comparison of deformed shapes with published solutions. (a) Symmetric solution. (b) Anti-symmetric solution.

$$
\mu_{\text {brk }}=0.5 . P=1.4 \mathrm{MN} \text {. }
$$

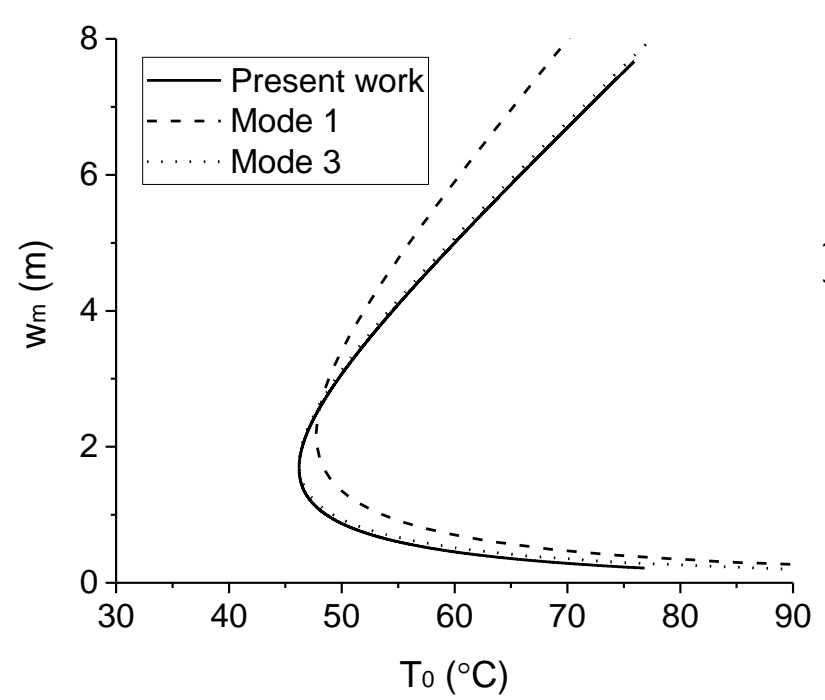

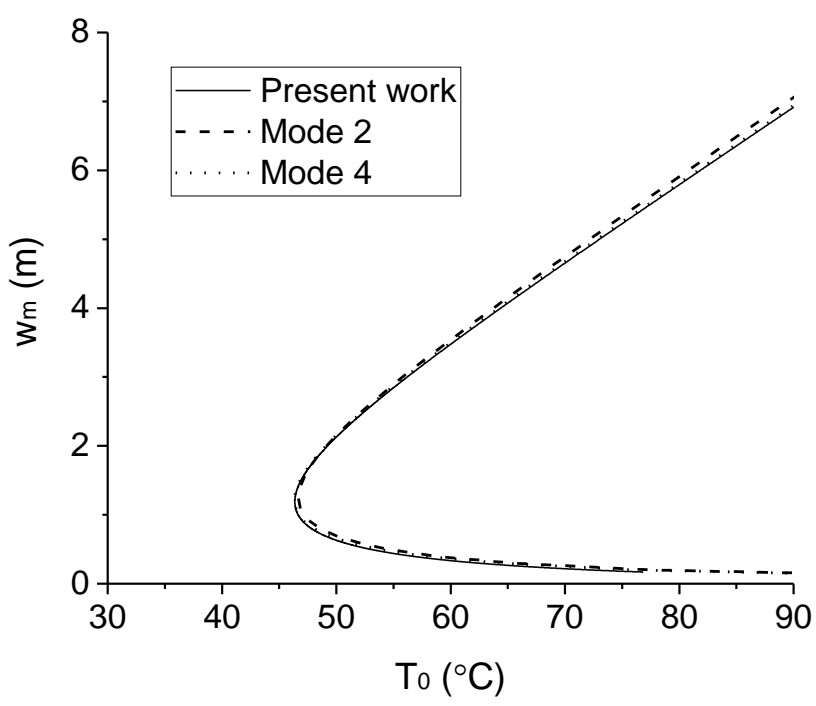


Fig. 9 Comparison of buckling paths with published solutions. (a) Symmetric solution. (b) Anti-symmetric solution. $\mu_{b r k}=$ $0.5 . P=1.4 \mathrm{MN}$.

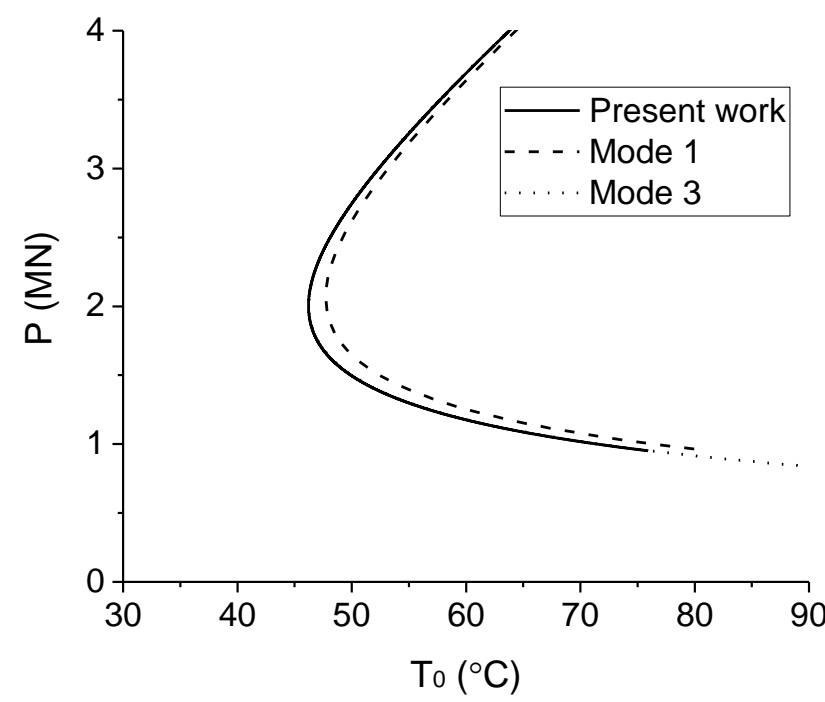

(a)

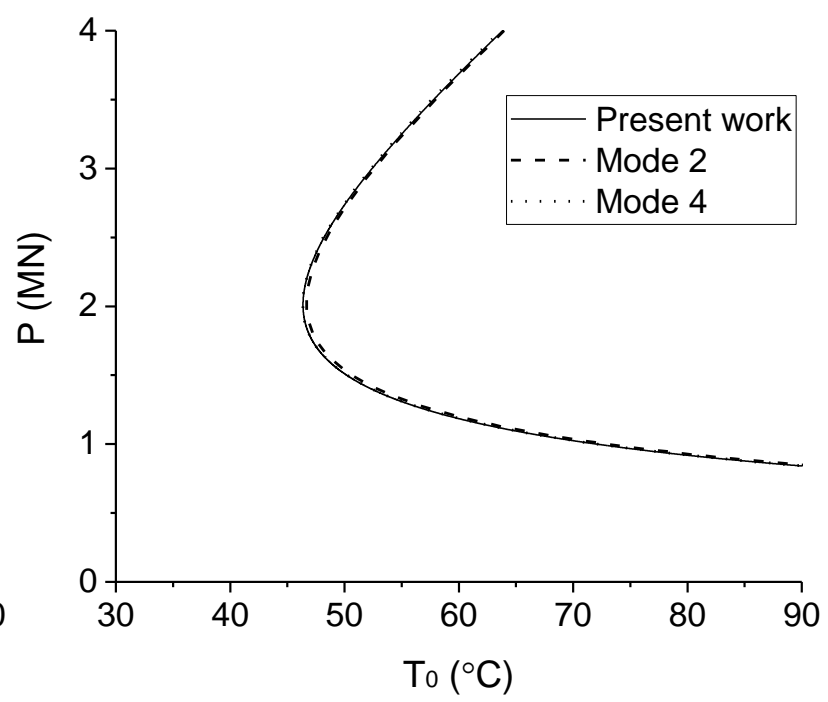

(b)

Fig. 10 Comparison of axial compressive force with published solutions. (a) Symmetric solution. (b) Anti-symmetric solution. $\mu_{b r k}=0.5 . P=1.4 \mathrm{MN}$.

\subsection{Energy analysis of a typical buckling path}

The typical relationship between localised lateral buckling amplitude $w_{m}$ and total temperature difference $T_{0}$ for a localised solution is shown in Fig. 11. This figure is for the symmetric solution (and $\mu_{b r k}=0.8$ ), but a very similar diagram is obtained for the anti-symmetric solution (Fig. 17 shows curves for both symmetric and anti-symmetric solutions). Two significant points $m$ and $e$ along the post-buckling path correspond to two critical temperature differences, namely the minimum critical temperature difference $T_{m}$ and the critical temperature difference $T_{c r} . T_{c r}$ is the temperature difference corresponding to the critical axial compressive force $P_{c r}$ and is obtained from Eq. (15). $T_{m}=48.2734{ }^{\circ} \mathrm{C}$ and $T_{c r}=$ 192.297493 ${ }^{\circ} \mathrm{C}$ for this case. In Fig. 11, two branches exist in the typical response of pipeline localised lateral buckling, which will be referred to as $m-b$ and $m-c$. The total potential energy of the buckled pipeline (in the mobilised region $0 \leq$ $x \leq l_{s}$ ) is given by

$$
\mathrm{V}=\frac{1}{2} E I \int_{0}^{L}\left(\frac{d^{2} w}{d x^{2}}\right)^{2} d x+\int_{0}^{L} h w(x) d x+\int_{0}^{l_{s}} f_{A} u(x) d x+\frac{1}{2 E A} \int_{0}^{l_{s}} P_{1}(x)^{2} d x
$$

where

$$
P_{1}(x)=P+f_{A} x
$$

while the total potential energy of the straight pipeline, namely before buckling, is given by

$$
V_{i}=\frac{1}{2 E A} \int_{0}^{l_{s}} P_{0}^{2} \mathrm{~d} x
$$

When $T_{0}$ is lower than $T_{m}$ only the trivial state exists and no localised lateral buckling occurs. The pipeline remains straight. However, when $T_{0}$ is larger than $T_{m}$, two localised lateral buckling states exist. Take $T_{0}=60^{\circ} \mathrm{C}$, for example. When $T_{0}$ reaches $60^{\circ} \mathrm{C}$, the pipeline will keep straight without disturbance or imperfection, corresponding to the point $\mathrm{d}$ in Fig. 11. If disturbance is imposed on the straight pipeline, the pipeline will jump to the localised buckling state $\mathrm{b}$ or state $\mathrm{c}$. The total energy corresponding to the localised buckling state is calculated through Eq. (33), which is used to analyse the relative stability of the two branches. Since $l_{s}$ depends on the precise shape of the solution, energies $\mathrm{V}$ for different solutions 
are not directly comparable. For a meaningful comparison we ensured pipes had equal length by adding extra length of (axially strained) pipe as necessary. The total energy of branches $m-b$ and $m-c$ for the localised post-buckling state are denoted by $V_{b}$ and $V_{c}$, respectively. $V_{i b}$ and $V_{i c}$ are the total potential energies of the straight pipeline of corresponding length $l_{s}$. $V_{b} / V_{i b}$ and $V_{c} / V_{i c}$ are illustrated in Fig. 12. We see that all the values of $V_{b} / V_{i b}$ are less than those of $V_{c} / V_{i c}$, which means that the branch $m-b$ is more stable than branch $m$-c. In addition, the value of $V_{c} / V_{i c}$ decreases to 1 with the increase of temperature difference, while all the values of $V_{c} / V_{i c}$ are larger than 1, which means that branch $m$-c is less stable than the trivial solution. The value of $V_{b} / V_{i b}$ also decreases with the increase of temperature difference, which means that the branch $m$ - $b$ becomes more stable with the increase of temperature difference. $V_{b} / V_{i b}=1$ when the temperature difference reaches $T_{e}=50.72^{\circ} \mathrm{C}$. For $T_{0}<T_{e}, V_{b} / V_{i b}$ is bigger than 1 , which means that the trivial solution is more stable. For $T_{0}>T_{e}$, $V_{b} / V_{i b}$ is smaller than 1 , which means that the branch $m-b$ is more stable than the trivial state. We conclude that the pipeline is likely to jump from the trivial branch onto branch $m-b$ under a sufficiently large disturbance when $T_{0}$ is larger than $T_{m}$. When $T_{0}$ increases to $T_{c r}$, the pipeline will suffer localised lateral buckling even if no disturbance exists.

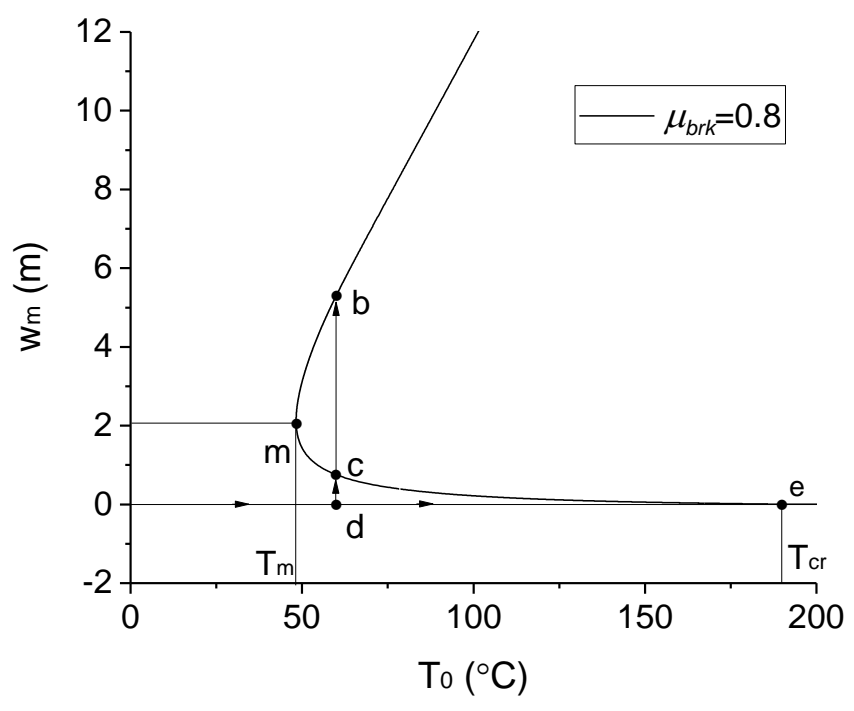

Fig. 11 Typical buckling path.

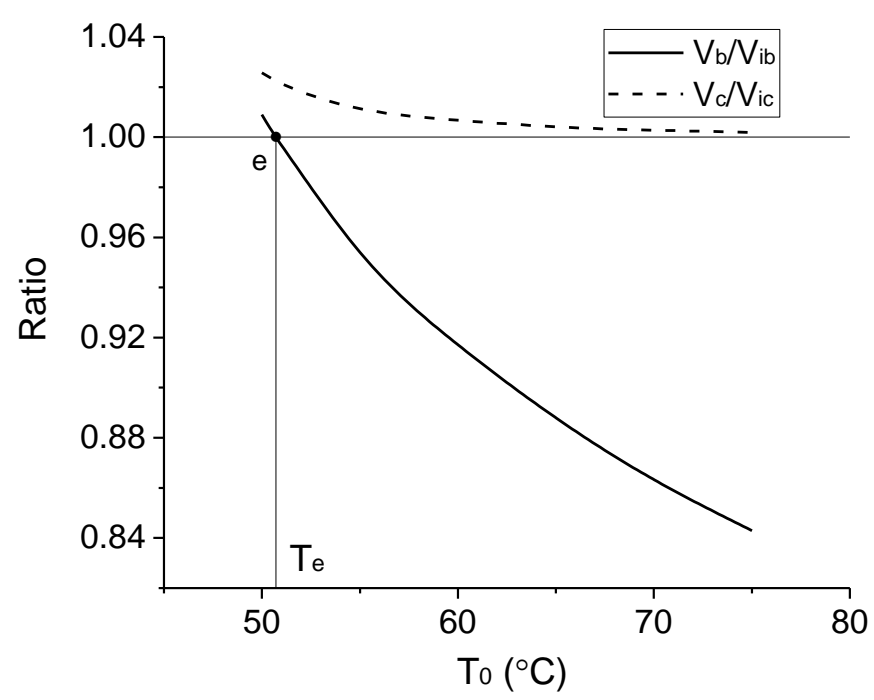

Fig. 12 The ratio of the energy between the buckled state and the pre-buckling state.

\subsection{Effect of nonlinear soil resistance on localised lateral buckling}

In this section, the effect of nonlinear soil resistance on localised lateral buckling is studied. First, the deformed shapes and bending stresses along the pipeline under different temperatures are analysed and discussed. Then, the influence of nonlinear 
soil resistance with different breakout resistances on localised lateral buckling behaviour is illustrated and discussed. Furthermore, the components of the maximum compressive stress are analysed in detail.

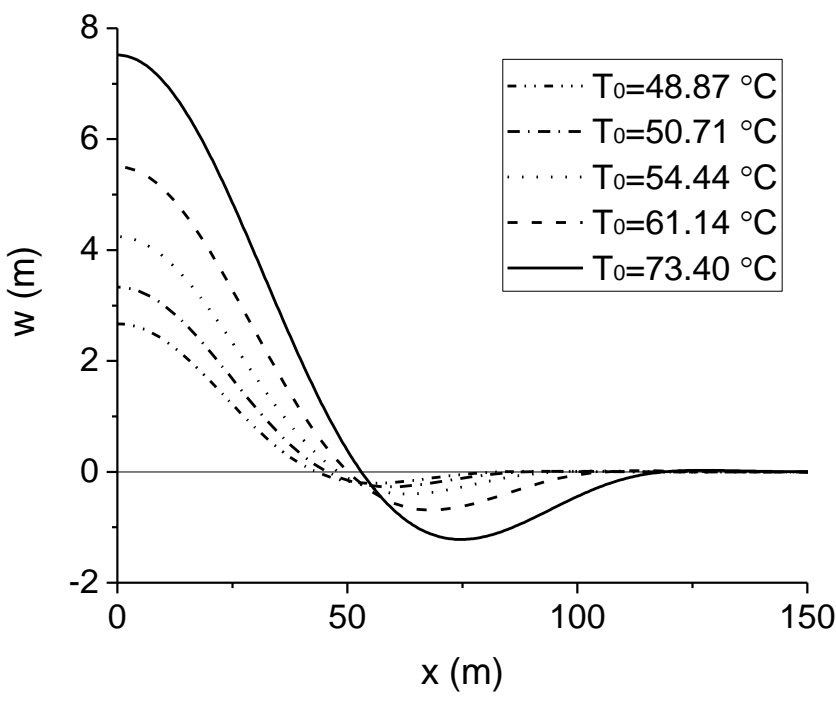

(a)

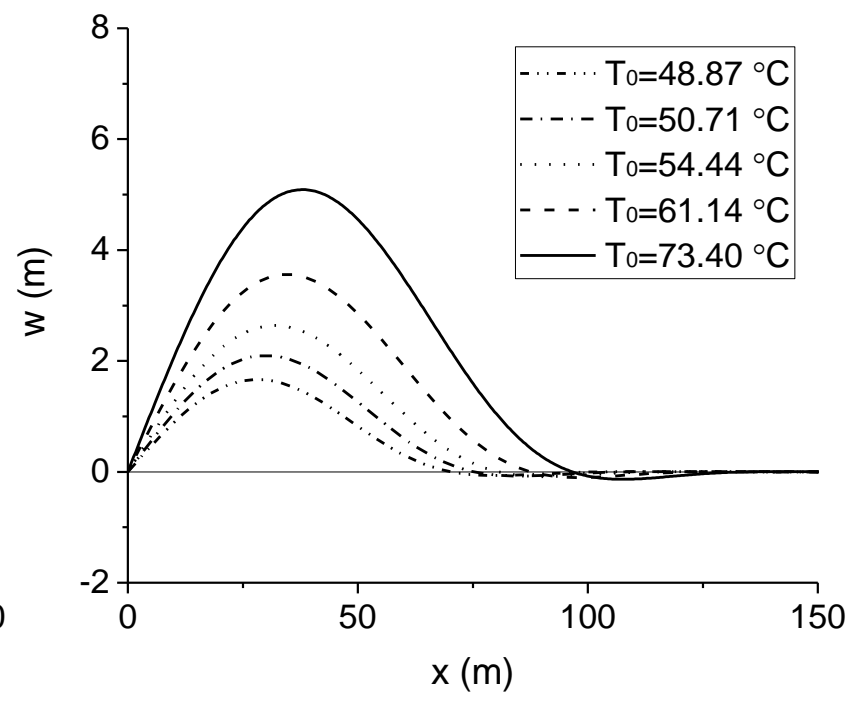

(b)

Fig. 13 Deformed shapes under different temperature differences. (a) Symmetric solution. (b) Anti-symmetric solution.

$$
\mu_{\text {brk }}=0.8 \text {. }
$$

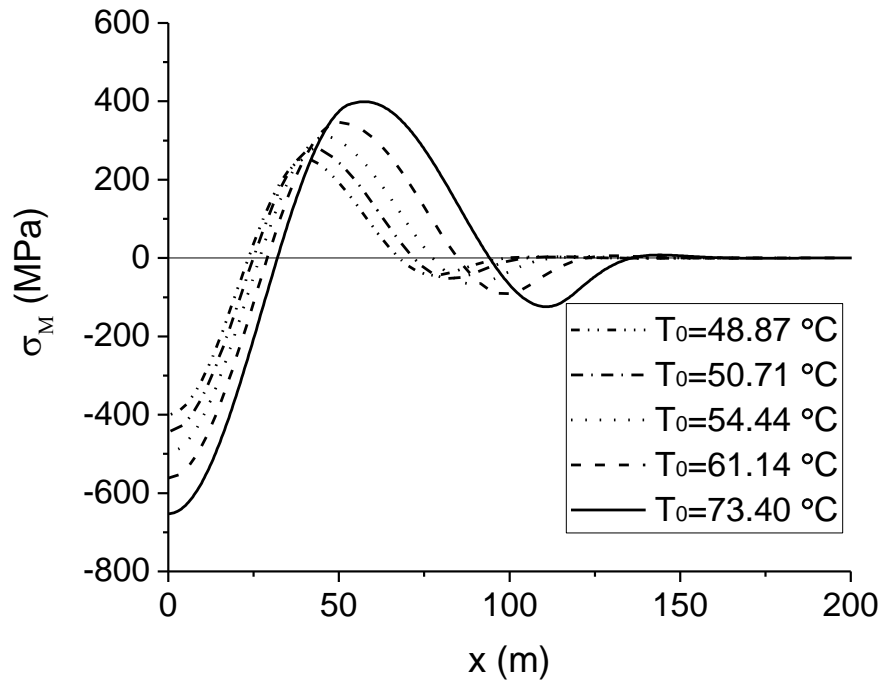

(a)

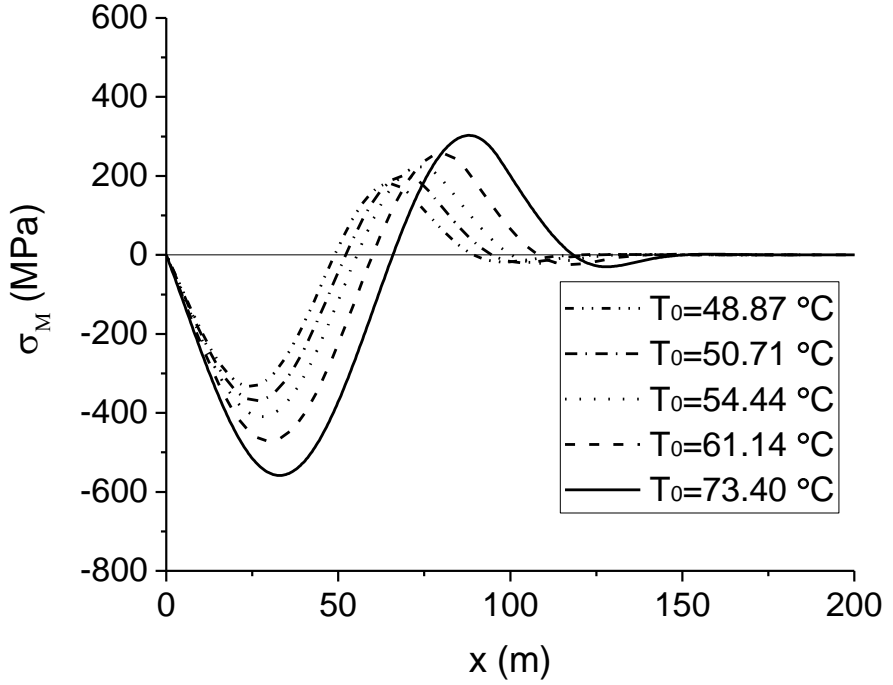

(b)

Fig. 14 Bending stresses under different temperature differences. (a) Symmetric solution. (b) Anti-symmetric solution.

$$
\mu_{b r k}=0.8 .
$$

The deformed shapes and the corresponding bending stress $\sigma_{M}=E D w_{3} / 2$ along the buckled pipeline under different temperature differences are presented in Fig. 13 and Fig. 14, respectively. In Fig. 13, it is obvious that a localised buckled shape is formed within a limited region in the middle of the pipeline due to the axial compressive force induced by temperature difference, which consists of half a primary lobe in the positive direction and a secondary lobe in the negative direction for half a buckled pipeline. There are many smaller lobes beyond these two lobes, but their lateral deformation is not significant for the present parameters. With the increase of temperature, both the buckled region and the lateral deflection increase for both the primary lobe and the secondary lobe, as shown in Fig. 13. Also, the amplitude of the bending stress is larger for higher temperature differences, as shown in Fig. 14. Moreover, the bending stress in the secondary lobe becomes larger too. Thus, the buckled pipeline becomes more dangerous with the increase of temperature difference. 


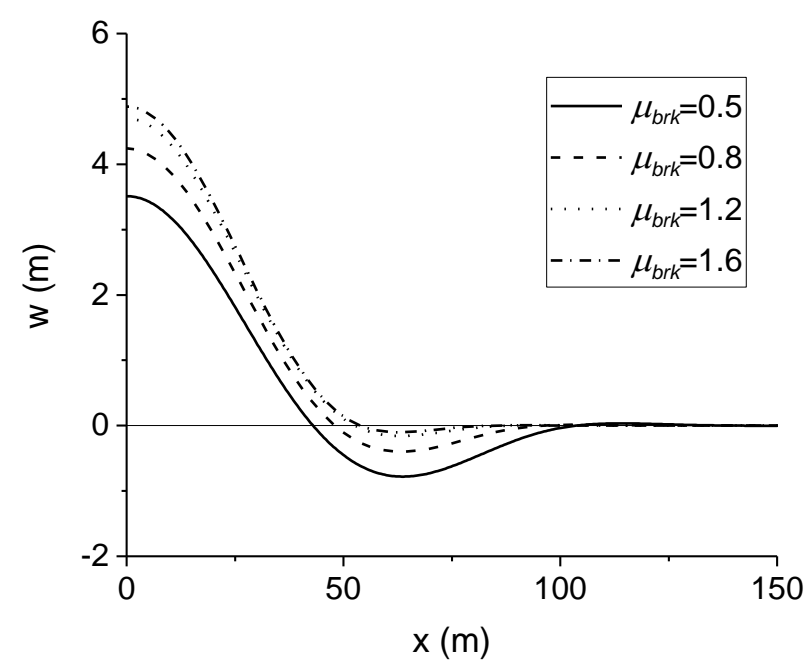

(a)

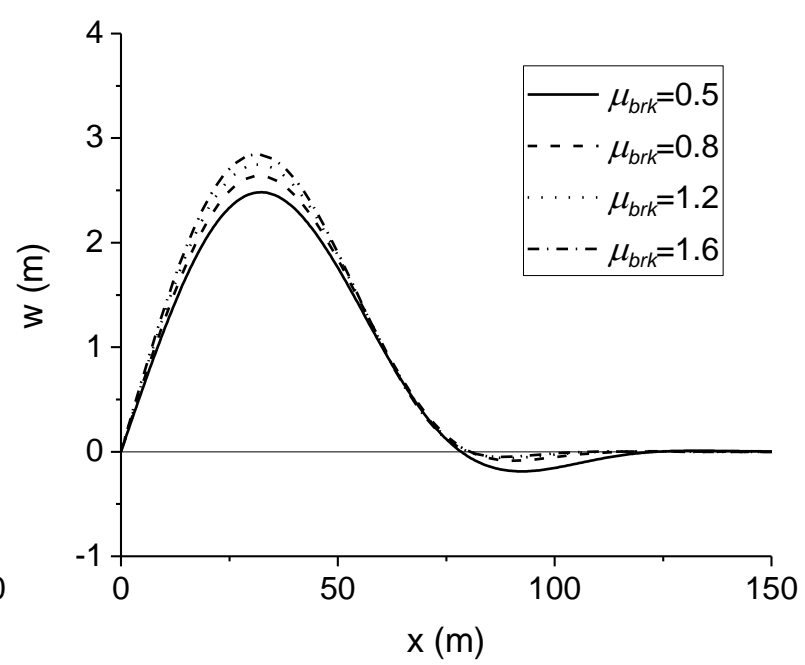

(b)

Fig. 15 Deformed shapes for different breakout resistances. (a) Symmetric solution. (b) Anti-symmetric solution. $P=$ 1.4 MN.

Fig. 15 illustrates the deformed shapes of partially embedded pipelines with different breakout resistances under the same axial compressive force $P=1.4 \mathrm{MN}$. It is clear that the breakout resistance has great influence on the deformed shapes of localised lateral buckling. The primary lobe for the case of $\mu_{b r k}=\mu_{r e s}=0.5$ is smaller than that for all the cases of $\mu_{b r k}>$ $\mu_{r e s}$ and increases with increasing $\mu_{b r k}$, while the secondary lobe decreases with increasing $\mu_{b r k}$. For the symmetric solution, the deformed shape becomes more similar to the deformed shape of mode 1 with the increase of the breakout resistance, which means that mode 1 is closer to the realistic buckling shape of partially embedded subsea pipelines with larger breakout resistance. Maybe this is the reason why mode 1 is mainly used to predict lateral buckling behaviour of partially embedded pipelines for symmetric solutions (DNV-RP-F110, 2007). For the anti-symmetric solution, the deformed shape becomes more similar to the deformed shape of mode 2 with the increase of the breakout resistance.

Fig. 16 illustrates the bending stress $\sigma_{M}$ of partially embedded pipelines at different breakout resistances under the same axial compressive force $P=1.4 \mathrm{MN}$. The amplitudes of bending stresses in the positive and negative direction increase with the increase of the breakout resistance. Thus, it is more dangerous for partially embedded pipelines with larger breakout resistance when localised lateral buckling happens.

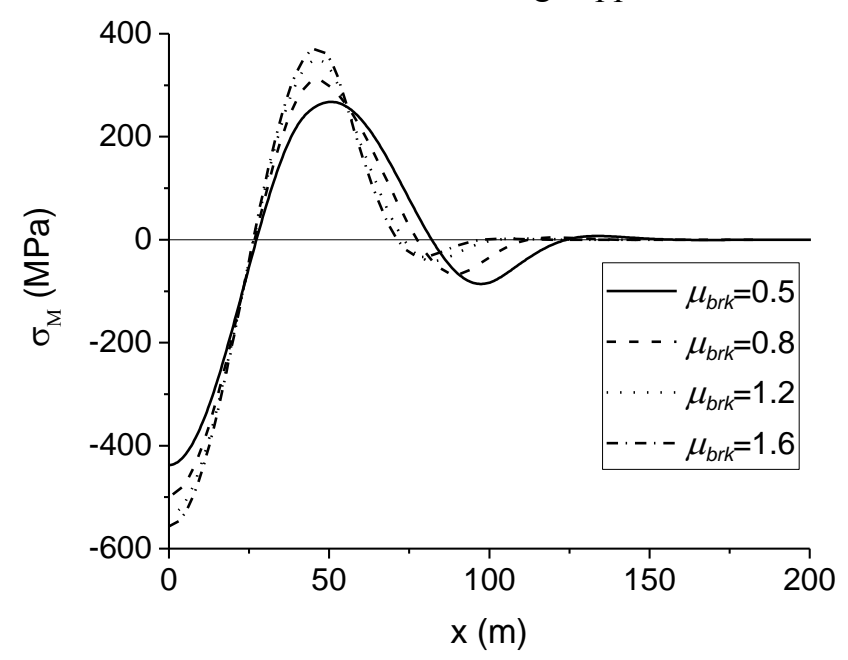

(a)

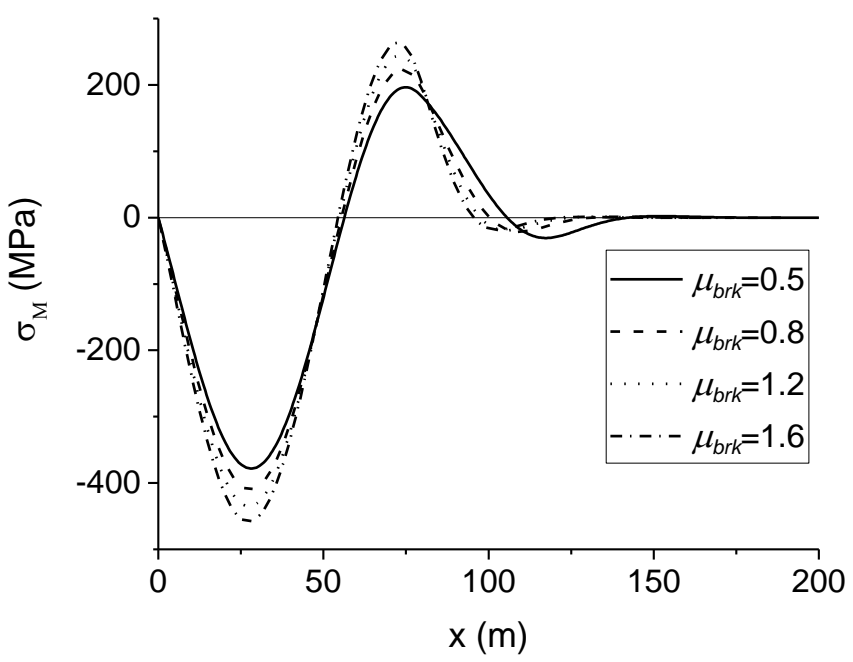

(b)

Fig. 16 Bending stresses for different breakout resistances. (a) Symmetric solution. (b) Anti-symmetric solution. $P=$ 1.4 MN. 


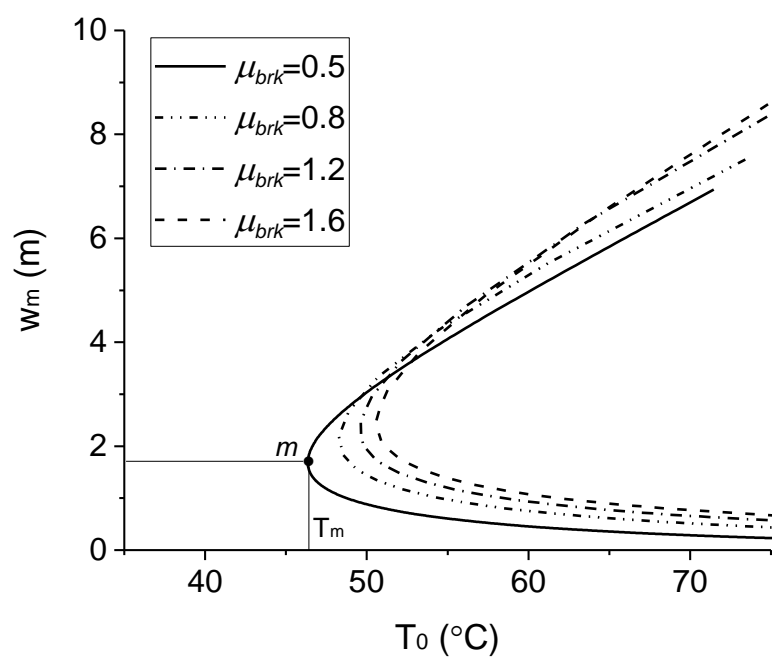

(a)

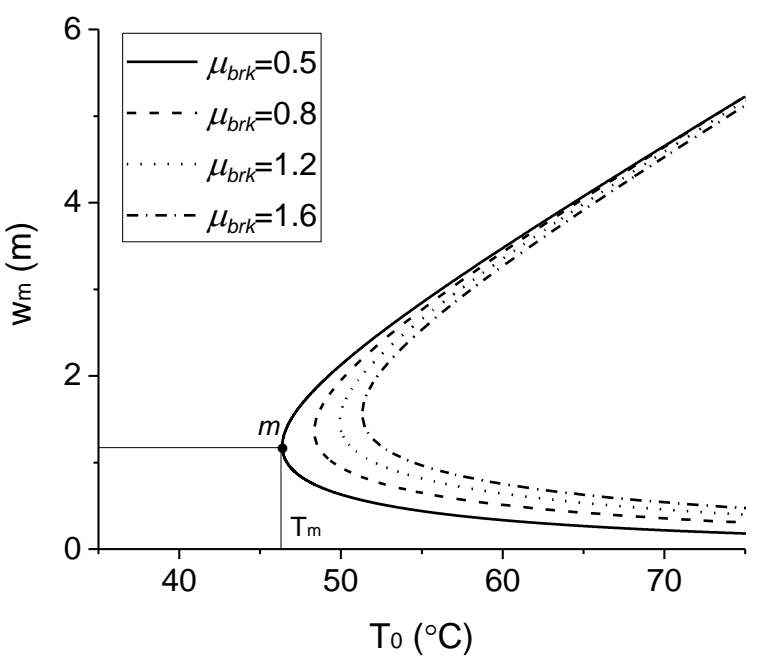

(b)

Fig. 17 The buckling path for different breakout resistances. (a) Symmetric solution. (b) Anti-symmetric solution.

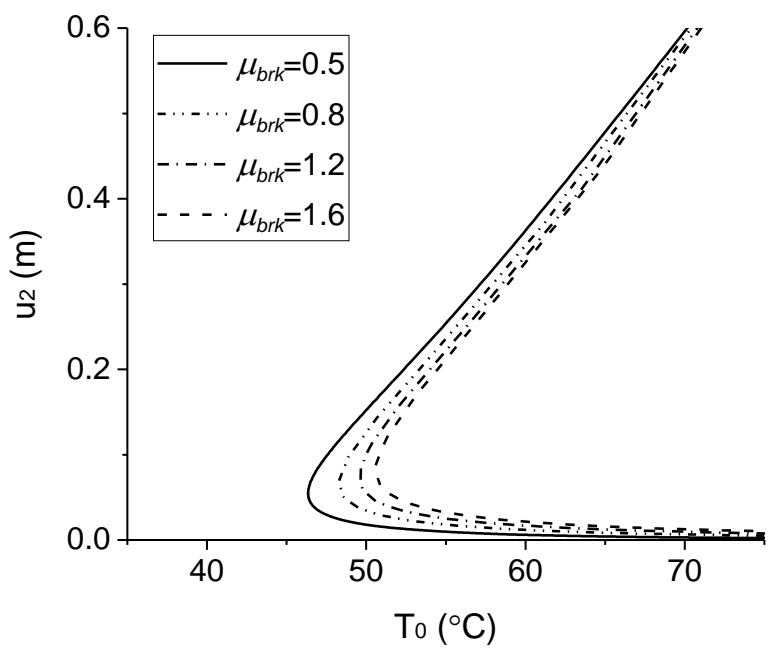

(a)

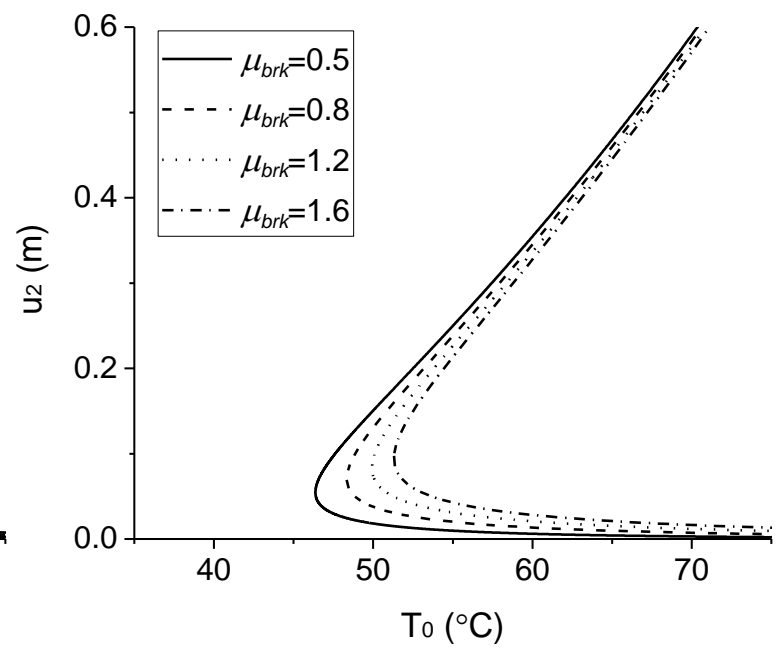

(b)

Fig. 18 The axial feed-in length $u_{2}$ for different breakout resistances. (a) Symmetric solution. (b) Anti-symmetric solution.

The buckling paths, namely the relationships between lateral buckling amplitude $w_{m}$ and total temperature difference $T_{0}$, at different breakout resistances are shown in Fig. 17, while the relationships between axial feed-in length $u_{2}$ and total temperature difference $T_{0}$ for different breakout resistances are shown in Fig. 18. A significant point $m$ along the postbuckling path corresponds to the minimum critical temperature difference $T_{m}$, which may be called an upper bound to the safe temperature. The minimum critical temperature difference $T_{m}$ increases with increasing breakout resistance, which means that it will be more difficult to have localised lateral buckling for pipelines with larger breakout resistance. After localised lateral buckling happens, the lateral buckling amplitude $w_{m}$ increases with the increase of the total temperature difference for a specific breakout resistance, as shown in Fig. 17. However, the rate of increase of the lateral buckling amplitude is smaller for smaller breakout resistances. In Fig. 18, the axial feed-in length $u_{2}$ increases with the increase of the total temperature difference for a specific breakout resistance. The axial feed-in length $u_{2}$ is smaller for larger breakout resistances under the same total temperature difference, while the lateral buckling amplitude increases faster for larger breakout resistance. The reason for this is that larger breakout resistance makes the deformation concentrate on the primary lobe to make the lateral buckling amplitude increase faster in spite of smaller $u_{2}$, as shown in Fig. 15. 


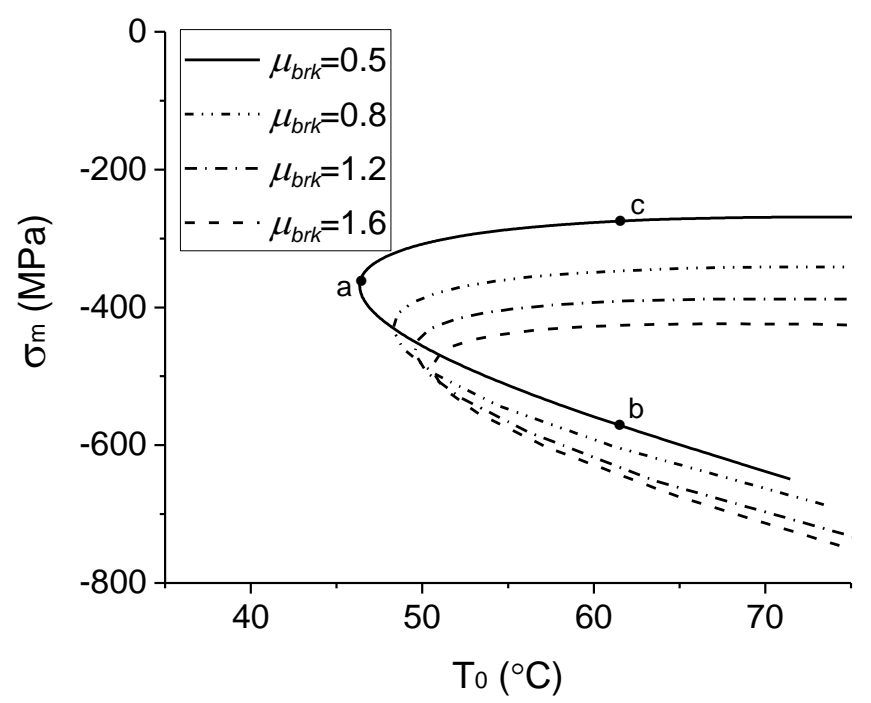

(a)

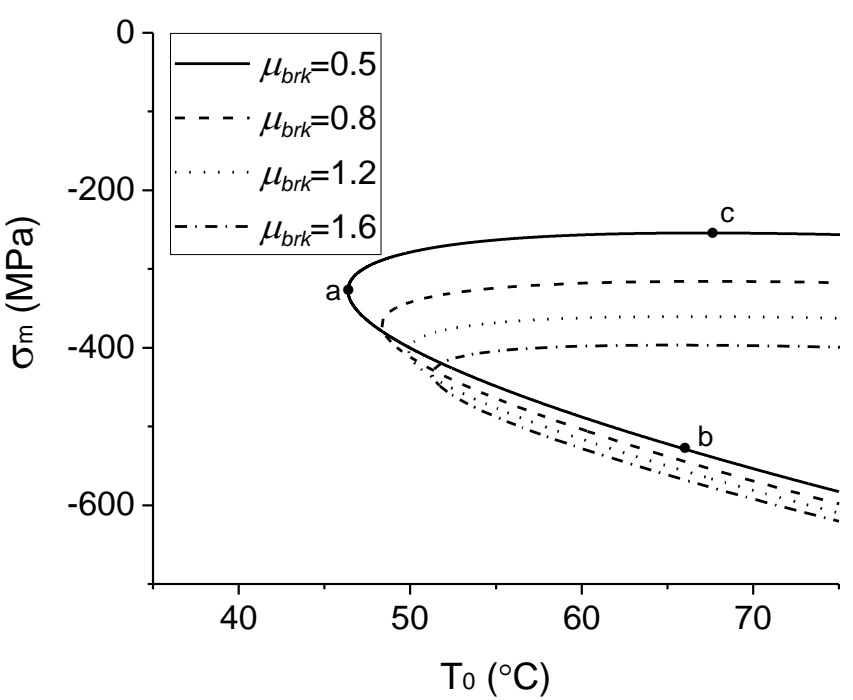

(b)

Fig. 19 The relationships between the maximum stress $\sigma_{m}$ and the operating temperature difference $T_{0}$. (a) Symmetric solution. (b) Anti-symmetric solution.

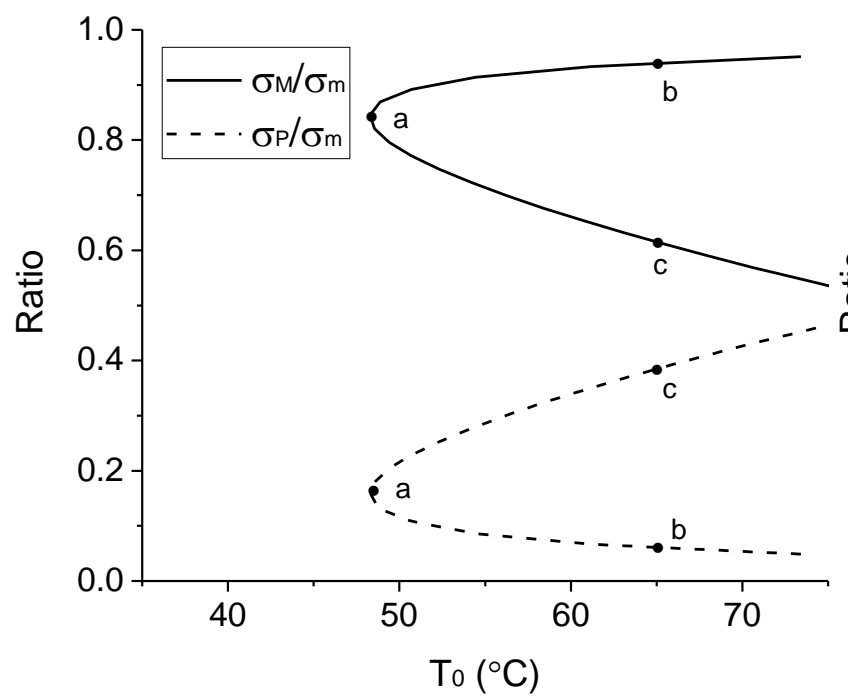

(a)

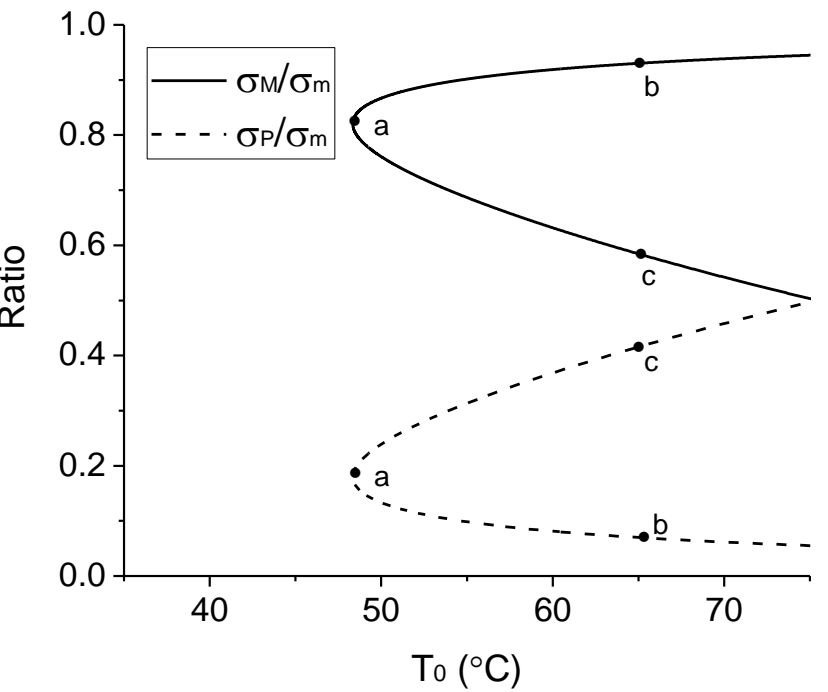

(b)

Fig. 20 The component of the maximum stress $\sigma_{m}$. (a) Symmetric solution. (b) Anti-symmetric solution. $\mu_{b r k}=0.8$.

The relationships between the maximum stress $\sigma_{m}$ and the operating temperature difference $T_{0}$ for different breakout resistances are shown in Fig. 19. The maximum stress consists of two parts, namely the bending stress $\sigma_{M}$ induced by bending moment and the axial compressive stress $\sigma_{P}=P / A$ due to the post-buckling axial compressive force $P$. The ratios between these two parts and the maximum stress $\sigma_{m}$ are shown in Fig. 20. We recall from the energy analysis in Section 3.3 that branch a-b is relatively stable while branch a-c is relatively unstable. In Fig. 19, the maximum stress $\sigma_{m}$ increases with the increase of the temperature difference for a specific breakout resistance. Also, for larger breakout resistance, the maximum stress $\sigma_{m}$ is larger under the same temperature difference, as shown in Fig. 19. So it is more dangerous for a pipeline with larger breakout resistance under the same temperature difference. According to Fig. 20, over $80 \%$ of the maximum stress $\sigma_{m}$ is induced by the bending moment. The stress ratio induced by the bending moment becomes lager with the increase of the temperature difference. 


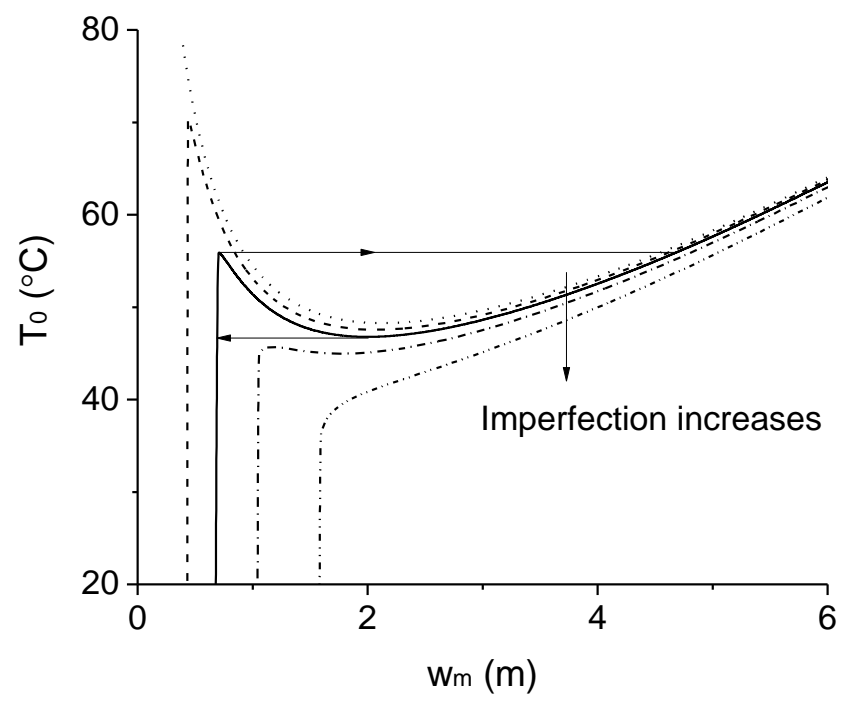

Fig. 21 The effect of imperfections on the load-deflection behaviour of Fig. 11. Arrows indicate dynamic jumps under increasing (to the right) or decreasing (to the left) $T_{0}$.

\section{Conclusions}

We have studied localised thermal buckling of pipelines by considering genuinely localised homoclinic solutions of the governing equations that bifurcate from a Hamiltonian-Hopf bifurcation. Curves of lateral deflection against temperature difference were obtained by using compatibility between axial and lateral deformation to relate the temperature difference to the axial compressive force in the pipe. Our focus has been on the nonlinear effect of breakout resistance on this loaddeflection behaviour of the buckled partially embedded pipeline for which we used the latest pipe-soil interaction model. Both symmetric and anti-symmetric buckling modes have been considered.

These localised solutions are often neglected but automatically display the decaying oscillatory behaviour with opposite lobes seen in real subsea pipelines. We have shown that this oscillatory behaviour is governed by the eigenvalues of the trivial straight solution, which can be obtained explicitly in terms of the physical parameters of the problem. No extra assumptions (for instance about imperfections or concentrated forces) have to be made. Decay rates and wavelengths of the localisation vary as parameters are varied and we have presented a parameter study in which we varied the temperature difference and a parameter characterising the breakout resistance of the surrounding soil. The effect of other parameters in the pipe-soil interaction model could similarly be studied.

The only assumption we make is that of a constant compressive force $\bar{P}$ in the localised solution, an assumption generally made in the literature. However, we make this assumption only in computing the shape of the localised solution and not in the computation of the corresponding temperature difference (based on deformational compatibility) and not in the energy analysis.

The energy analysis reveals several critical temperatures in addition to the Hamiltonian-Hopf critical temperature $T_{\mathrm{cr}}$. No localised solutions exist for temperatures less than $T_{m}$, which therefore represents an upper bound to safe operating temperatures for the pipeline. For temperatures larger than $T_{m}$ two symmetric and two anti-symmetric localised solutions are available. Initially these have larger energy than the straight unbuckled pipe, but we find that, typically, for only slightly higher temperatures ( $T>T_{e}$ ) one of the symmetric and one of the anti-symmetric localised solutions acquires an energy lower than that of the straight solution (see Fig. 12). For such temperatures, the unbuckled pipe, although still linearly stable, can therefore be considered unstable under sufficiently large perturbations (e.g., dynamic disturbances due to irregular fluid flow through the pipe or earthquakes).

Because of imperfections, the trivial branch d-e in Fig. 11 may not be followed exactly. Possible sources of imperfection include: non-straightness of the unstressed pipe, variations in the thickness of the pipe and unevenness of the supporting seabed. Since the Hamiltonian-Hopf bifurcation at $T_{\mathrm{cr}}$ is subcritical, localised buckling of the pipeline is in fact extremely 
sensitive to such imperfections. The effect of imperfections on the load-deflection behaviour of the pipeline is illustrated in Fig. 21, which shows an enlargement of the region of interest in Fig. 11 with some typical imperfection curves added. For these imperfection curves we used the modified version

$$
T_{0}=\frac{\left(P+\sqrt{2 E A f_{A}\left(u_{2}-u_{20}\right)}\right)}{E A \alpha}
$$

of Eq. (15) with only the vertical lines added by hand. The imperfection $u_{20}$ can be interpreted as the horizontal shortening due to a stressed or unstressed local non-straightness of the pipe. Fig. 21 is qualitatively similar to load-deflection diagrams in (Taylor and Gan, 1986b). We see that for some values of the imperfection the load-deflection curves have folds where dynamic jumps of the structure may occur under both increasing and decreasing temperature.

We compare in the Appendix our safe upper temperature bound $T_{\mathrm{m}}$ with formulae recommended in DNV-RP-F110 for design checks on the possibility of lateral buckling triggered by imperfections.

In some cases the imperfection behaviour may be used to induce controlled buckling of the pipeline in order to avoid high levels of axial expansion, for instance by inserting buoyancy sections that locally reduce the submerged weight of the pipe (Li et al., 2016). Other methods for initiating lateral buckling by means of imperfection are discussed in (Sinclair et al., 2009). Our results in Fig. 17 and Fig. 18 suggest that reducing the breakout resistance could be part of such buckling initiation strategies.

From our parameter studies the following conclusions can be drawn:

(i) The deformed shape and the buckling path can be predicted accurately by using the assumption of mode 3 and mode 4 (in Hobbs's classification) when the lateral soil resistance is constant. However, the assumption of mode 1 and mode 2 will overestimate the lateral displacement amplitude.

(ii) For a specific nonlinear lateral soil resistance, under increasing temperature, both the buckled region and the lateral deflection increase for both the primary lobe and the secondary lobe of the deformed shape. The amplitude of the bending stress is larger for higher temperature difference.

(iii) The breakout resistance has a great influence on the deformed buckling shape. Under increasing breakout resistance the primary lobe becomes bigger, while the secondary lobe becomes smaller. The deformed shape becomes more similar to that of mode 1 for a symmetric solution and to that of mode 2 for an anti-symmetric solution under increasing breakout resistance.

(iv) The minimum critical temperature difference $T_{m}$ increases with increasing breakout resistance. After localised lateral buckling happens, the rate of increase of lateral buckling amplitudes is smaller for smaller breakout resistances. However, the axial feed-in length $u_{2}$ is smaller for larger breakout resistances under the same total temperature difference.

(v) The maximum stress $\sigma_{m}$ is composed of bending stress $\sigma_{M}$, induced by the bending moment, and axial compressive stress $\sigma_{P}$, due to the post-buckling axial compressive force. Over $80 \%$ of the maximum stress $\sigma_{m}$ is induced by bending moment after localised lateral buckling happens. Thus, the key point to control the maximum stress in the pipeline is to control the bending moment by controlling the deformed shape of the buckled pipeline. The maximum stress $\sigma_{m}$ increases with the increase of the temperature difference. For larger breakout resistance, the maximum stress $\sigma_{m}$ is larger under the same temperature difference. So it is more dangerous for a pipeline with larger breakout resistance.

\section{Acknowledgments}

The authors would like to acknowledge that the work described in this paper was funded by the National Key Basic Research Program of China (2014CB046805).

\section{Appendix}

The design code DNV-RP-F110 (Section 6.3.3) gives the following formulae, based on Hobbs's analysis, for checks on the possibility of localised buckling triggered by imperfections: 


$$
S_{\infty}=2.29 \frac{E I}{\bar{L}^{2}}, \quad \bar{L}=\left(\frac{(E I)^{3}}{f_{L}^{2} E A}\right)^{0.125}
$$

where $E I$ is the bending stiffness, $A$ is the cross-sectional area of the pipe and $f_{\mathrm{L}}$ is a lower bound for the lateral soil resistance. Here $S_{\infty}$ is the 'lateral global buckling capacity', i.e., the lower limit of the axial force that may activate lateral buckling.

For the parameters in Table 1 this gives for the corresponding temperature $T_{\infty}=\frac{S_{\infty}}{E A \alpha}$ the value $T_{\infty}=54.77{ }^{\circ} \mathrm{C}$, if we take $f_{L}=\mu_{\mathrm{L}} W_{\text {pipe }}$ with $\mu_{\mathrm{L}}=0.5$ (i.e., equal to $\mu_{\mathrm{A}}$ ). $T_{\infty}$ should be compared with our upper safe temperature limit $T_{\mathrm{m}}$. In Fig. 11 , for $\mu_{\text {brk }}=0.8$, we find $T_{\mathrm{m}}=48.2734{ }^{\circ} \mathrm{C}$, while Fig. 17 and Fig. 18 show that $T_{\mathrm{m}}$ decreases with decreasing values of $\mu_{\text {brk }}$. This suggests that for soils with low breakout resistance the recommendations in DNV-RP-F110 may be too optimistic.

\section{References}

Bruton, D., White, D., Cheuk, C., Bolton, M. and Carr, M., 2006. Pipe/soil interaction behaviour during lateral buckling, including large-amplitude cyclic displacement tests by the Safebuck JIP, Offshore Technology Conference, OTC-17944MS.

Champneys, A.R. and Spence, A., 1993. Hunting for homoclinic orbits in reversible systems: A shooting technique. Adv. Comput. Math., 1(1): 81-108.

Chatterjee, S., White, D.J. and Randolph, M.F., 2012. Numerical simulations of pipe-soil interaction during large lateral movements on clay. Geotechnique, 62(8): 693-705.

Cheuk, C.Y., White, D.J. and Bolton, M.D., 2007. Large-scale modelling of soil-pipe interaction during large amplitude cyclic movements of partially embedded pipelines. Can. Geotech. J., 44(8): 977-996.

Croll, J.G.A., 1997. A simplified model of upheaval thermal buckling of subsea pipelines. Thin-Walled Struct., 29(1-4): 59-78.

Dingle, H.R.C., White, D.J. and Gaudin, C., 2008. Mechanisms of pipe embedment and lateral breakout on soft clay. Can. Geotech. J., 45(5): 636-652.

DNV-RP-F110, 2007. Global buckling of submarine pipelines structural design due to high temperature/high pressure. Det Norske Veritas.

Hobbs, R.E., 1981. Pipeline buckling caused by axial loads. J. Constr. Steel Res., 1(2): 2-10.

Hobbs, R.E., 1984. In-service buckling of heated pipelines. J. Transp. Eng., 110(2): 175-189.

Hong, Z., Liu, R., Liu, W. and Yan, S., 2015a. A lateral global buckling failure envelope for a high temperature and high pressure (HT/HP) submarine pipeline. Appl. Ocean Res., 51: 117-128.

Hong, Z., Liu, R., Liu, W. and Yan, S., 2015b. Study on lateral buckling characteristics of a submarine pipeline with a single arch symmetric initial imperfection. Ocean Eng., 108: 21-32.

Hunt, G.W., Bolt, H. and Thompson, J., 1989. Structural localization phenomena and the dynamical phase-space analogy. Proceedings of the Royal Society of London A: Mathematical, Physical and Engineering Sciences, 425: 245-267.

Hutchinson, J. and Koiter, W., 1970. Postbuckling theory. Appl. Mech. Rev., 23(12): 1353-1366.

Karampour, H. and Albermani, F., 2014. Experimental and numerical investigations of buckle interaction in subsea pipelines. Eng. Struct., 66: 81-88.

Karampour, H., Albermani, F. and Gross, J., 2013. On lateral and upheaval buckling of subsea pipelines. Eng. Struct., 52: 317-330.

Karampour, H., Albermani, F. and Major, P., 2015. Interaction between lateral buckling and propagation buckling in textured deep subsea pipelines. ASME 2015 34th International Conference on Ocean, Offshore and Arctic Engineering, OMAE2015-41013.

Karampour, H., Albermani, F. and Veidt, M., 2013. Buckle interaction in deep subsea pipelines. Thin-Walled Struct. 72: $113-120$

Kerr, A.D., 1978. Analysis of thermal track buckling in the lateral plane. Acta Mechanica, 30: 17-50. 
Lagrange, R. and Averbuch, D., 2012. Solution methods for the growth of a repeating imperfection in the line of a strut on a nonlinear foundation. Int. J. Mech. Sci., 63(1): 48-58.

Lambrakos, K., 1985. Marine pipeline soil friction coefficients from in-situ testing. Ocean Eng., 12(2): 131-150.

Leckie, S.H.F., Draper, S., White, D.J., Cheng, L. and Fogliani, A., 2015. Lifelong embedment and spanning of a pipeline on a mobile seabed. Coastal Eng., 95: 130-146.

Leckie, S.H.F., Mohr, H., Draper, S., McLean, D.L., White, D.J. and Cheng, L., 2016. Sedimentation-induced burial of subsea pipelines: Observations from field data and laboratory experiments. Coastal Eng., 114: 137-158.

Li, G., Zhan, L. and Li, H., 2016. An analytical solution to lateral buckling control of subsea pipelines by distributed buoyancy sections. Thin-Walled Struct., 107: 221-230.

Liu, R., Liu, W., Wu, X. and Yan, S., 2014. Global lateral buckling analysis of idealized subsea pipelines. J. Cent. South Univ., 21(1): 416-427.

Lyons, C.G., 1973. Soil resistance to lateral sliding of marine pipelines. Offshore Technology Conference, OTC-1876MS.

Randolph, M.F. and White, D.J., 2008. Pipeline embedment in deep water: Processes and quantitative assessment, Offshore Technology Conference, OTC-19128-MS.

Shi, R., Wang, L., Guo, Z. and Yuan, F., 2013. Upheaval buckling of a pipeline with prop imperfection on a plastic soft seabed. Thin-Walled Struct., 65: 1-6.

Sinclair, F., Carr, M., Bruton, D. and Farrant, T., 2009. Design challenges and experience with controlled lateral buckle initiation methods, ASME 2009 28th International Conference on Ocean, Offshore and Arctic Engineering, OMAE200979434.

Sumer, B.M., Truelsen, C., Sichmann, T. and Fredsøe, J., 2001. Onset of scour below pipelines and self-burial. Coastal Eng., 42(4): 313-335.

Taylor, N. and Gan, A.B., 1986a. Refined modelling for the lateral buckling of submarine pipelines. J. Constr. Steel Res., 6(2): 143-162.

Taylor, N. and Gan, A.B., 1986b. Submarine pipeline buckling-imperfection studies. Thin-Walled Struct., 4(4): $295-323$.

Taylor, N. and Gan, A.B., 1987. Refined modelling for the vertical buckling of submarine pipelines. J. Constr. Steel Res., 7(1): 55-74.

Taylor, N. and Tran, V., 1993. Prop-imperfection subsea pipeline buckling. Mar. Struct., 6(4): 325-358.

Taylor, N. and Tran, V., 1996. Experimental and theoretical studies in subsea pipeline buckling. Mar. Struct., 9(2): 211257.

van der Heijden, G.H.M., Champneys, A.R. and Thompson, J.M.T., 1998. The spatial complexity of localized buckling in rods with noncircular cross section. SIAM J. Appl. Math., 59(1): 198-221.

Wagner, D.A., James, D.M., Harald, B. and Olav, S., 1989. Pipe-soil interaction model. Journal of Waterway, Port, Coastal and Ocean Engineering, 115(2): 205-220.

Wang, L., Shi, R., Yuan, F., Guo, Z. and Yu, L., 2011. Global buckling of pipelines in the vertical plane with a soft seabed. Appl. Ocean Res., 33(2): 130-136.

White, D.J. and Cheuk, C.Y., 2008. Modelling the soil resistance on seabed pipelines during large cycles of lateral movement. Mar Struct, 21(1): 59-79.

Zeng, X. and Duan, M., 2014. Mode localization in lateral buckling of partially embedded submarine pipelines. Int. J. Solids Struct., 51(10): 1991-1999.

Zhu, J., Attard, M.M. and Kellermann, D.C., 2015. In-plane nonlinear localised lateral buckling of straight pipelines. Eng, Struct., 103: 37-52. 